\title{
DESIGN OF SOFT COMPOSITE FINGER WITH ADJUSTABLE JOINT STIFFNESS
}

\author{
A Thesis \\ by \\ ZARYAB SHAHID \\ Submitted to the Office of Graduate and Professional Studies of \\ Texas A\&M University \\ in partial fulfillment of the requirements for the degree of \\ MASTER OF SCIENCE
}

Chair of Committee, Seok Chang Ryu

Committee Members, Anastasia Muliana

Michael Moreno

Head of Department, Andreas A. Polycarpou

August 2018

Major Subject: Mechanical Engineering

Copyright 2018 Zaryab Shahid 


\begin{abstract}
This research presents the design of a soft composite finger with tunable joint stiffness. The com-posite finger, made from two different types of silicone, has hybrid actuation principle combining tendon and pneumatic actuation schemes. Tendons control the finger shape in a prescribed di-rection to demonstrate discrete bending behavior due to different material moduli, similar to the human finger's discrete bending. Whereas, pneumatic actuation changes the stiffness of joints us-ing air chambers. The feasibility of adjustable stiffness joints is proven using both the parallel spring-damper model and experiments, demonstrating the stiffening effect when pressurized. A set of experiments were also conducted on fingers with four different chamber designs to see the effect of chamber shape on stiffening and the discrete bending capability of the finger. These stiff-ened fingers lead to firm grasp as they constrain the object better and apply higher grasping force. The gripper made up of soft composite fingers can grasp objects of various sizes, shapes and in different orientations.
\end{abstract}




\section{DEDICATION}

To the community of researchers working in the field of Soft Robotics.

To Scheherzad Alvi, I am really grateful to have you during this time. Thanks for your great moral support, continuous care and encouraging me to believe in myself. 


\section{ACKNOWLEDGMENTS}

I thank my family and friends for their support and cooperation throughout my graduate school. I also thank Dr. Seok Chang Ryu, my Advisor, for his mentoring, advices and valuable comments that helped me in completion and success of this study.

I would like to thank my colleagues in BioRobotics Laboratory, Texas A\&M University for logistical support during this research. Special thanks to Abigail Glatman and Immanuel Ponminissery, undergraduate researchers to help me in design and fabrication stages of this research. 


\section{CONTRIBUTORS AND FUNDING SOURCES}

\section{Contributors}

This work was supported by a thesis committee consisting of Assistant Professor Seok Chang Ryu and Professor Anastasia Muliana of the Department of Mechanical Engineering and Assistant Professor Michael Moreno of the Department of Biomedical Engineering.

All work for the thesis was completed by the student, under the advisement of Seok Chang Ryu of the Department of Mechanical Engineering.

\section{Funding Sources}

There are no outside funding contributions to acknowledge related to the research and compilation of this document. 


\section{NOMENCLATURE}

\section{PDMS}

$f_{o}$

$d_{i}$

$(\Delta d)_{i}$

$K_{i}$

$\Delta K_{i}$

$(\Delta f)_{i}$

$n$

$P$

V

$A_{O}$

$a_{i}$

$h$

$x_{i}$

$b_{i}$

$A_{\text {ini }}$

$A_{\text {fin }}$

$K_{\text {finger }}$

$\Delta K_{\text {finger }}$

$d_{\text {finger }}$

$\Delta d_{\text {finger }}$

$K_{s}$
Polydimethylsiloxane

Tendon force

Compression due to pneumatic actuation

Elongation of air spring due to pressurization

Stiffness constant of the linear spring

Additional stiffness constant of the air spring

Air spring force due to pressurization

Polytropic constant

Atmospheric pressure

Volume of air chamber

Cross-sectional area of air chamber

Length of semi-major axis of the ellipse

Height of the finger

Change in lateral side of the chamber due to pressurization

Length of semi-minor axis of the ellipse

Initial area of silicone around the joint

Final area of silicone around the joint after pressurization

Stiffness constant of the finger

Additional stiffness constant due to pressurization

Cumulative deformation in linear spring of both joints

Total elongation in both air springs due to pressurization

Stiffness of Non-linear spring 
$\Delta e$

$N$

$f$

$f_{s}$

$\alpha$

$\tau_{o}$

$m_{o}$

$\ddot{x}_{2}$

$g$

$a$
Deformation of the finger

Normal force

Friction force

Friction force when the robotic arm is stationary

Rate of increase of shear strength

Shear strength of the surface force when load is zero

Mass of the object

Acceleration of the object at the tip

Object gravity vector

Radius of contact area 
TABLE OF CONTENTS

Page

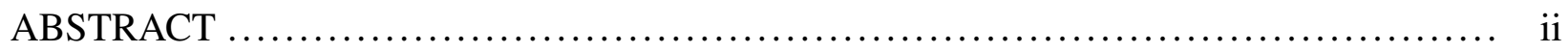

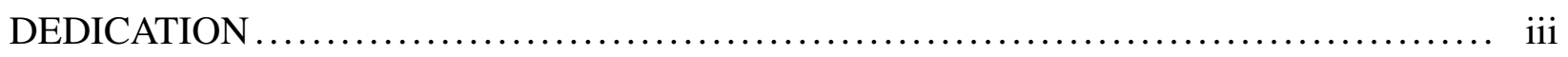

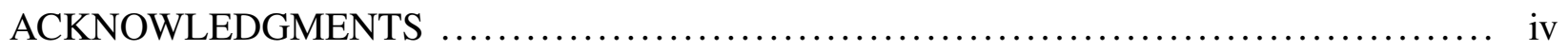

CONTRIBUTORS AND FUNDING SOURCES $\ldots \ldots \ldots \ldots \ldots \ldots \ldots \ldots \ldots \ldots \ldots \ldots \ldots, \mathrm{v}$

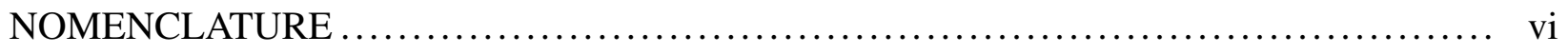

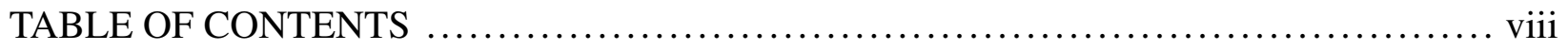

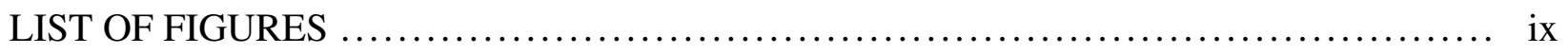

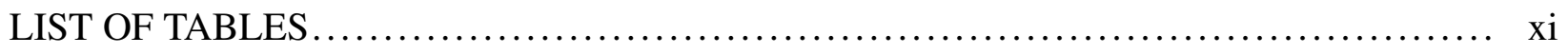

1. INTRODUCTION AND LITERATURE REVIEW $\ldots \ldots \ldots \ldots \ldots \ldots \ldots \ldots \ldots \ldots \ldots \ldots \ldots \ldots \ldots \ldots \ldots \ldots \ldots$

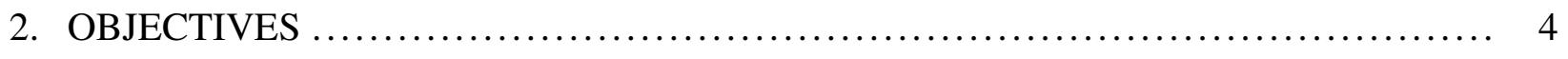

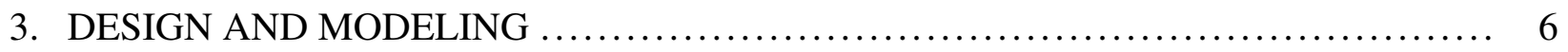

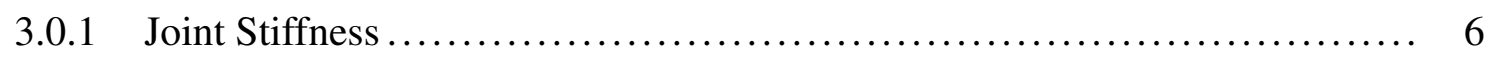

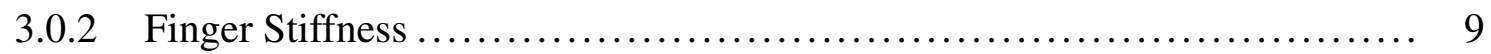

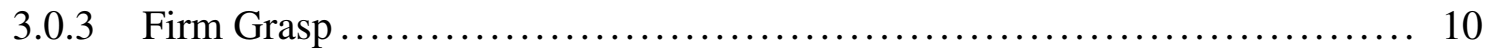

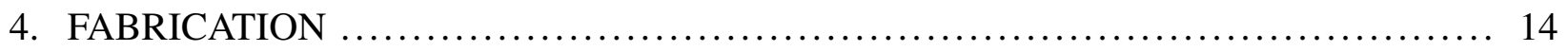

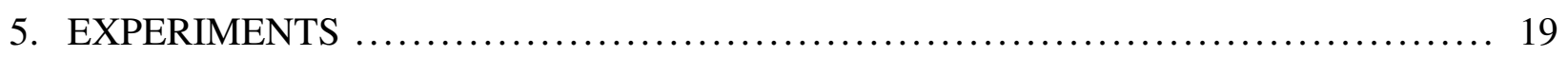

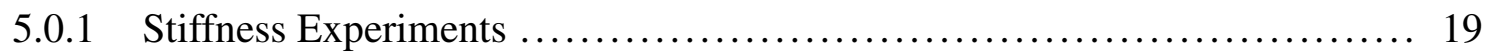

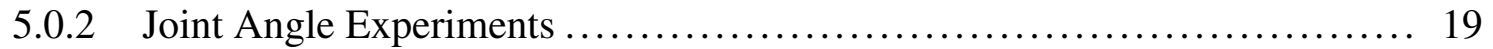

5.0 .3 Firm grasp Experiments ....................................... 20

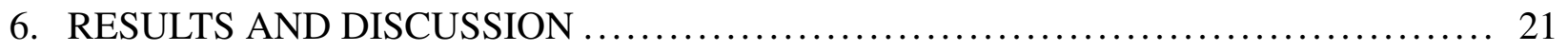

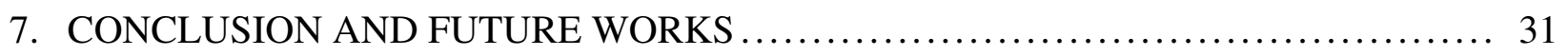

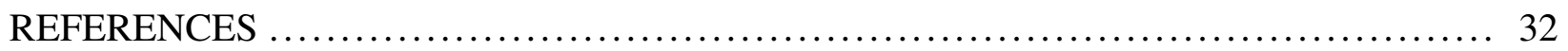




\section{LIST OF FIGURES}

FIGURE

Page

1.1 Design of soft composite finger: (a) robotic hand made up of three soft composite fingers holding a fragile daily-life object. (b) section view of pressurized chamber in the joint. (c) soft composite finger similar to human finger.

3.1 Modeling of stiffening joints: (a) composite finger showing geometrical parameters with deformed ellipse. Shape change due to pneumatic actuation is shown by red dashed line. Shape change due to tendon actuation is shown by black dashed line. (b) $t_{i}$, thickness of soft silicone around the joint. $A_{i n i}$ represents the area of silicone around the joint before pressurization. (c) $x_{i}$, change in lateral sides of the air chamber due to pressurization. (d) pressure acting on rectangular cross-sectional area. ...............................................................

3.2 Increasing pressure stiffens the joints: (a) proximal joint (b) distal joint ............

3.3 Soft composite finger with augmented structural rigidity to better constrain the object at multiple points. The inextensible tendon keeps AA' constant, whereas B'B and CC' increases with pressurization which exerts a push against the object... 12

3.4 Modeling of normal force between soft finger tip and object.

4.1 Fabrication of soft composite finger. (a) Apparatus consists of 3-D printed mold, 2 metal tubes, silicone tube, and 2 knuckles. (b) Metal tubes are inserted into the tendon channels, then silicone tubing is inserted into the pneumatic channel and then knuckles are inserted to secure silicone tubing. (c) Filled PDMS mold. (d) Cured PDMS structure removed from the mold. (e) PDMS structure is inserted into ECOFLEX mold. (f) ECOFLEX mold is prepared as explained earlier (b). (g) Filled ECOFLEX mold. (h) Cured ECOFLEX removed from the mold. (i) Knuckles are sealed using a shallow tray filled with PDMS. (j) Soft composite finger with silicone tubing and Kevlar thread routed.

4.2 Different finger designs. (a) Vertical Ellipse (b) Circle (c) Small Circle (d) Horizontal Ellipse.

5.1 Experimental setups for soft composite finger. (a) Experimental setup for stiffness tests using force sensor and motorized linear module. (b) Soft composite finger showing discrete bending behavior. 
6.1 Both experimental results (connected by dashed lines) and model predictions (connected by solid lines) show that soft composite finger with different chamber shapes stiffens when the chambers are pressurized at constant pulling lengths of $3 \mathrm{~mm}$, $7 \mathrm{~mm}, 12 \mathrm{~mm}$. (a) Vertical ellipse (b) Circle. As expected, the model of the vertical ellipse satisfying all underlying assumptions better predicts the experimental results. 24

6.2 Both experimental results (connected by dashed lines) and model predictions (connected by solid lines) show that soft composite finger with different chamber shapes stiffens when the chambers are pressurized at constant pulling lengths of $3 \mathrm{~mm}$, $7 \mathrm{~mm}, 12 \mathrm{~mm}$. (a) Small circle (b) Horizontal ellipse ....................... 25

6.3 The soft composite finger shows discrete bending behavior when the tendons are pulled at constant pressures of $0 \mathrm{~Pa}, 6600 \mathrm{~Pa}, 10400 \mathrm{~Pa}$. (a) Vertical ellipse (proximal) (b) Vertical ellipse (distal) $\ldots \ldots \ldots \ldots \ldots \ldots \ldots \ldots \ldots \ldots \ldots \ldots \ldots \ldots \ldots \ldots \ldots \ldots \ldots \ldots \ldots \ldots, 26$

6.4 The soft composite finger shows discrete bending behavior when the tendons are pulled at constant pressures of $0 \mathrm{~Pa}, 6600 \mathrm{~Pa}, 10400 \mathrm{~Pa}$. (a) Circle (proximal) (b)

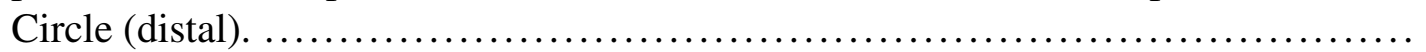

6.5 The soft composite finger shows discrete bending behavior when the tendons are pulled at constant pressures of $0 \mathrm{~Pa}, 6600 \mathrm{~Pa}, 10400 \mathrm{~Pa}$. (a) Smallcircle (proximal) (b) Smallcircle (distal).

6.6 The soft composite finger shows discrete bending behavior when the tendons are pulled at constant pressures of $0 \mathrm{~Pa}, 6600 \mathrm{~Pa}, 10400 \mathrm{~Pa}$. (a) Horizontal ellipse (proximal) (b) Horizontal ellipse (distal).

6.7 The designed gripper can grasp various size(diameter from $20 \mathrm{~mm}$ to $100 \mathrm{~mm}$ ) and shape objects such as circular, rectangular, oval, cylindrical, etc. at various orientations. (a) Robotic arm with gripper (b) Wine glass (c) Avocado (d) Wine glass grasped in a different orientation (e) Robotic arm with gripper actuated (f) Kevlar Spool (g) Fidget Spinner (h) Microcontroller board. 


\section{LIST OF TABLES}

TABLE

Page

6.1 Summarized results of firm grasp experiment on a Wine glass for two cases (Empty and with weights) 


\section{INTRODUCTION AND LITERATURE REVIEW}

The human finger is a state of the art composite structure in terms of design, compliance and manipulation capacity [1]. One of the most engrossing features of the human finger is its intricate joint design that defines its passive compliance and the direction of motion of the finger. The human finger has both multiple degrees of freedom and multi-directional compliance due to elastic elements (ligaments, tendons, geometry of bone cavity) in it along with overarching joint design [1], [2]. Also, this joint design helps the human finger to conform to unknown objects, thus enhancing its adaptive capacity. Inspired by the human hands, several robotic hands have been studied previously [3], [4]. Although, all these robotic hands have precise position control and produce strong forces at finger tips, they have fixed degrees of freedom due to simplified embedded mechanical joints. These kinds of joints have made these hands non-compliant and less adaptive to objects they manipulate. In addition, they cannot handle delicate objects such as soft body organs [5] without advanced haptics capability.

With the advent of Soft Robotics, researchers have developed controllable structures from elastomeric materials [6]. These structures can bend, expand, compress and twist to achieve the desired end effector motion. A pneumatic [7], tendon [8], hydraulic [9], shape memory materials [10], material jamming [11], or electro-active polymers [12] actuation scheme deform the shape and reinforce the structural strength. Due to the nature of inherent softness in both, the body material and actuation scheme, these robots can perform a variety of tasks in unstructured environments and can interact with human beings without causing any harm [13], [14]. Soft robotic hands have higher DOF and greater passive compliance [15], [16]. These less constrained joints allow more compliance, which enable these robotic hands to grab and manipulate objects [17]. Nevertheless, these entirely soft structures also lead to decreased grasping force followed by an issue of slipping of objects and making it hard to predict their deformed shapes [17], [18], [19].

Inspired by the varying stiffness and object grasping capability of the octopus tentacle [20], robotics researchers have figured out actuation schemes to tune the stiffness of soft structures. Ali 


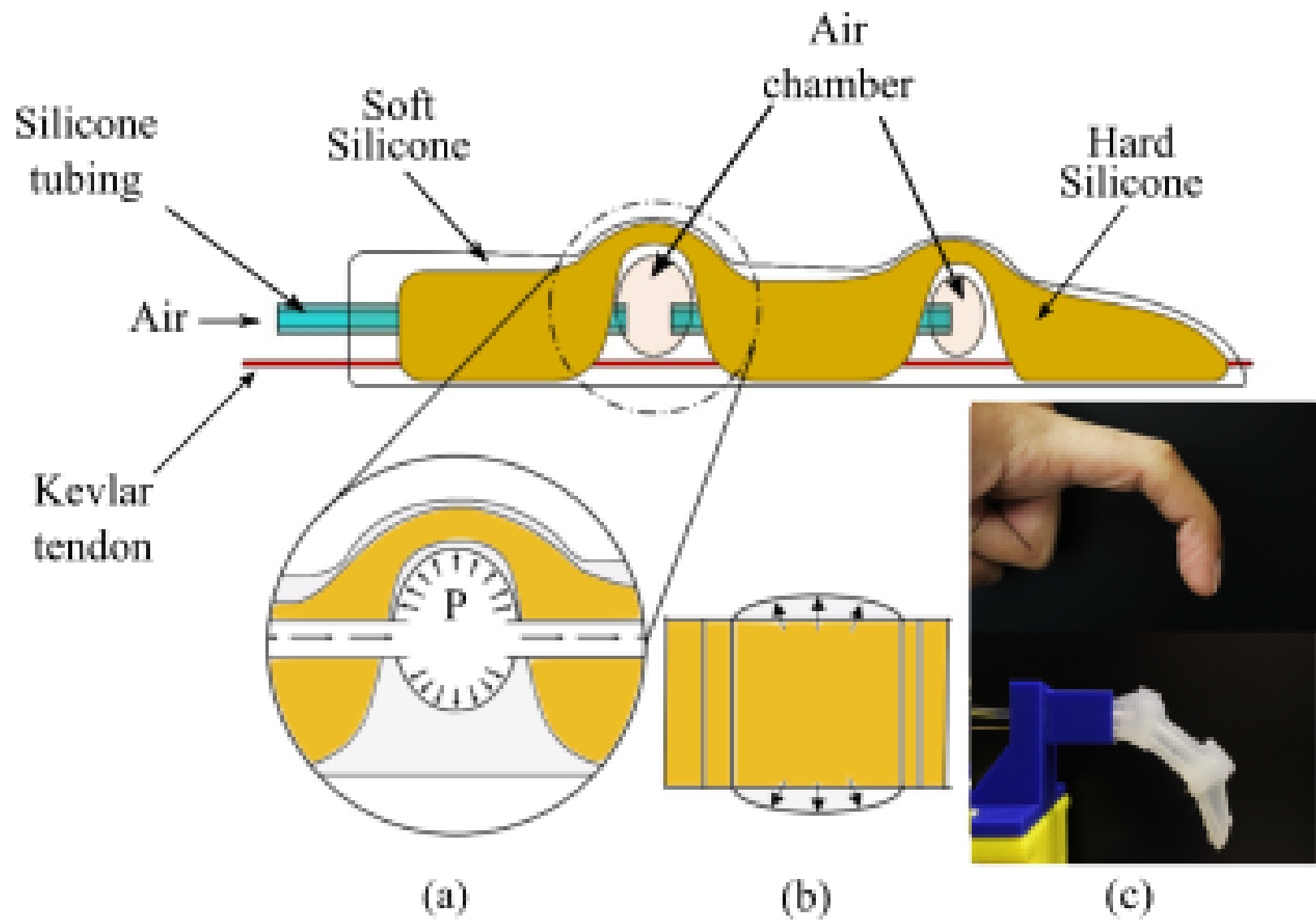

Figure 1.1: Design of soft composite finger: (a) robotic hand made up of three soft composite fingers holding a fragile daily-life object. (b) section view of pressurized chamber in the joint. (c) soft composite finger similar to human finger.

et al developed the stiffness-controllable robot arm with the hybrid and inherently antagonistic actuation scheme [21]. Stiffness variation is realized with tendons routed within the silicone body that can be actuated using stepper motors. A similar concept has been proposed in [22]. A tendondriven and pneumatic-actuated bio-inspired soft manipulator is presented which can change its length and control its stiffness. Researchers have further demonstrated the stiffening capability of soft robots by implementing the hybrid actuation approach that employs both tendon and pneumatic actuation [23]. Moreover, some other stiffness variation methods are also implemented in soft robots such as, using granules that can be jammed by applying a vacuum [24], [25], varying friction between the overlapping layers of Mylar film to tune stiffness [26] and thermally tunable composite that can achieve wide range of stiffness [27]. 
In this thesis, under the enlightenment of the physiological study of the human finger, we propose a potential approach of the soft composite structure with hybrid actuation for anthropomorphic soft fingers as shown in Fig.1.1. Motivated by [28], the composite structure integrates soft silicone and relatively rigid silicone to partially combine the merits of both materials. The hybrid actuation includes both tendon and pneumatic actuation. The tendon based actuation is implemented to bend the finger in a defined and predictable manner [29]. Whereas, the pneumatic actuation is used to stiffen the finger to achieve firm grasp [21], [23], [22].

The soft anthropomorphic soft composite finger is designed to have two joints: the distal joint and the proximal joint. These joints tune the compliance of the composite structure that could locally change the stiffness properties of the finger [5]. The asymmetric design of each joint enhances discrete bending as the top part of the joint consists of rigid silicone, whereas the bottom part is soft silicone. Also, the shape of the chamber affects the stiffness and bending capability of the finger. Such an approach will address the challenges in how to make fingers more compliant and adaptive to the significant range of objects, yet still perpetuate a sufficient amount of stiffness to enhance firm grasp in a predictable way.

This thesis is organized as follows: It includes the design of a soft composite finger with the stiffness model to support the feasibility of pressurized joints. The fabrication process is explained in Section II. To demonstrate the stiffening capability and discrete bending of the finger, several experiments have been conducted on fingers with different joint designs (see Section III). The results and discussion section (Section IV) compare different joint designs in terms of stiffness and bending behavior. Finally, the soft gripper is attached to one link robotic arm to experimentally validate firm grasp. The last section concludes this study by presenting future work. 


\section{OBJECTIVES}

The crux of this study is to deal with the feasibility of pressurizing joints to cause stiffness in the finger. A stiffness model is derived for the proposed finger design to validate that pressurizing of the joints causes stiffness in the finger. In addition to this, several experiments have been conducted to evaluate the performance of finger in terms of stiffness followed by measuring joint angle for both joints at an allowable pressure range to show explicit discrete bending behavior. This model and experimental study is also done for four different chamber shapes. Subsequently, these composite fingers are assembled into a gripper to substantiate that pressurizing the joints lead to firm grasp. These are the following objectives to justify our claims.

1. Design and Fabrication

- Joint Stiffness Model: derive stiffness model using the geometrical parameter of the joints.

- Finger Stiffness Model: derive the model for finger stiffness.

- Firm Grasp: show that stiffened fingers lead to firm grasp.

- Fabrication: explain fabrication procedure staring from raw materials to the final product.

2. Experimental Assessment

- Stiffness Evaluation: measure stiffness of the finger at different pressures for certain pulling lengths to validate that pressurization leads to stiffening.

- Joint Angle Experiments: measure joint angles for both distal and proximal joints at different pressures and pulling lengths to validate the discrete bending behavior.

- Firm Grasping: demonstrate firm grasp using a gripper attached to robotic arm to validate that stiffened fingers can have a firm grasp over the object. 
Given the above objectives, there are certain limitations to this study. Firstly, the composite finger shows viscoelastic behavior which is not taken into consideration when deriving the stiffness model of the finger. However, the experiments are repeated several times after substantial time to avoid the viscoelastic effect of the finger. 


\section{DESIGN AND MODELING}

\subsubsection{Joint Stiffness}

This section focuses on the analytical modeling of two joints to see the effect of pressurizing on stiffness of the joints. Each joint has a linear spring, damper and air spring fixed in a parallel combination (see Fig.3.1). When tendons are pulled, the joints are compressed so a linear spring is used to model the deformation in the joint. In addition, joints elongate in the longitudinal direction when they are pressurized so an air spring is used to model this behavior [30], [31]. The damper is added to model the viscoelastic effect of soft materials but our main focus is on modeling the stiffness effect. This model captures the explicit relationship between force due to pressurization and stiffness of the air spring by taking joint parameters into consideration. The stress-strain relationship for both silicones is highly non-linear but non-linearities of the material are not accounted in this model. Also, this model is limited to low values of input pressure and pulling lengths.

The constitutive relation for the parallel spring system in the joint can be written as

$$
\begin{gathered}
f_{o}=\left(K_{i}+\Delta K_{i}\right)\left(d_{i}-(\Delta d)_{i}\right) \\
\left\{\begin{array}{l}
i=d, \text { for distal joint } \\
i=p, \text { for proximal joint }
\end{array}\right.
\end{gathered}
$$

where $d_{i}$ is the deformation in the linear spring, $f_{o}$ is the tendon force, $(\Delta d)_{i}$ is the elongation of the air spring due to pressurization, $K_{i}$ is the stiffness constant of the linear spring, $\Delta K_{i}$ is the additional stiffness constant of the air spring.

The pressurization of composite finger is considered as polytropic process so the air spring force due to pressurization, $(\Delta f)_{i}$ can be related to deformation of air spring using the following relation [32]. 


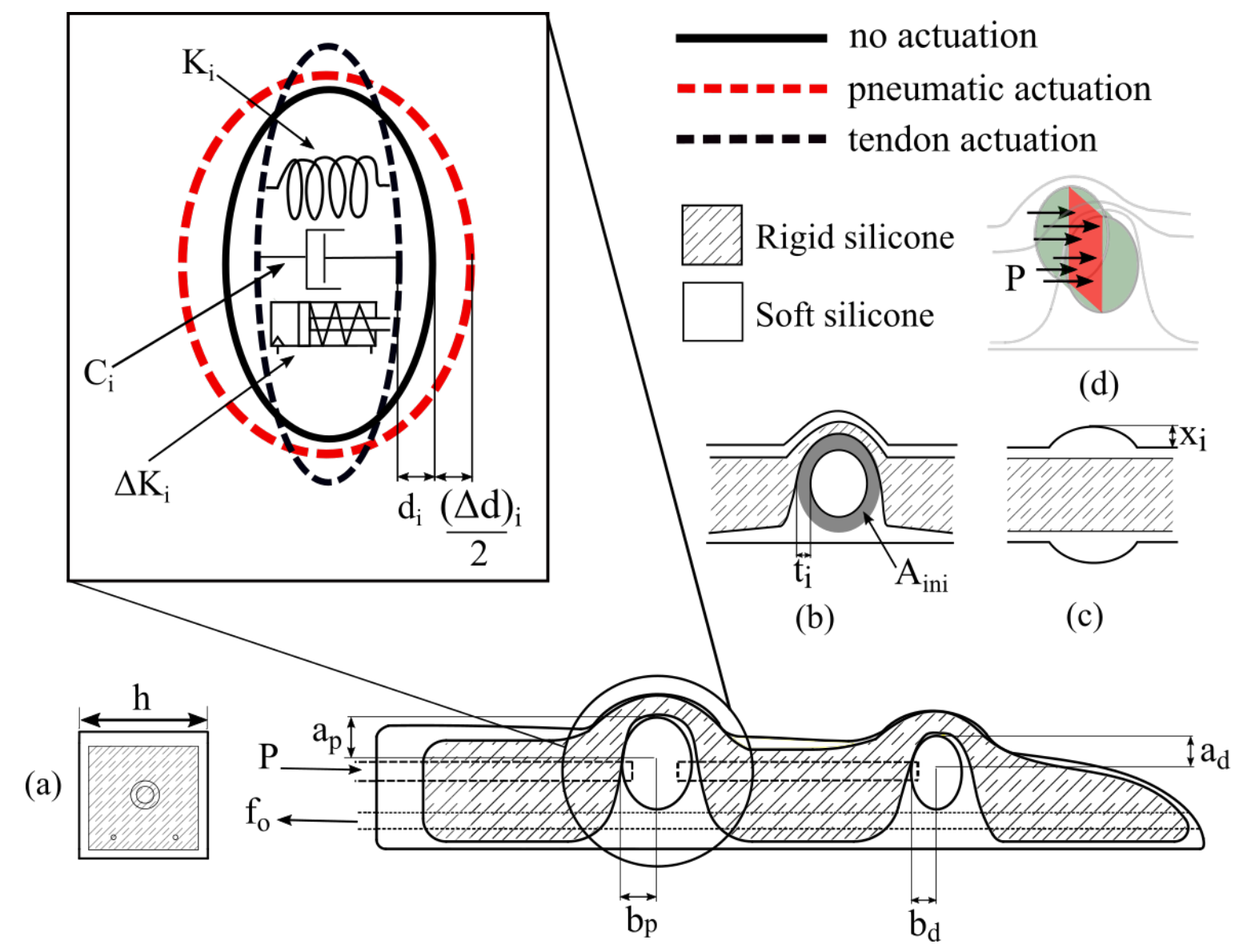

Figure 3.1: Modeling of stiffening joints: (a) composite finger showing geometrical parameters with deformed ellipse. Shape change due to pneumatic actuation is shown by red dashed line. Shape change due to tendon actuation is shown by black dashed line. (b) $t_{i}$, thickness of soft silicone around the joint. $A_{i n i}$ represents the area of silicone around the joint before pressurization. (c) $x_{i}$, change in lateral sides of the air chamber due to pressurization. (d) pressure acting on rectangular cross-sectional area.

$$
(\Delta f)_{i}=\frac{n P_{o} A_{o}^{2}(\Delta d)_{i}}{V}
$$

where, $n$ is equal to 1 because temperature is assumed constant during pressurization, $P_{o}$ is atmospheric pressure, $V$ is volume of air in the air chamber and $A_{o}$ is cross-sectional area of air chamber which is assumed as a rectangle in our case. The rectangular cross-sectional area plays significant role in determining the stiffening capability of the joint (see Fig.3.1(d)).

This model can be implemented on all the chamber shapes but we have chosen vertical ellipse 
for this case. We substituted expressions for $A_{o}$ and $V$ in Equation (3.2) to relate deformation of air spring with the joint parameters of the finger.

$$
(\Delta d)_{i}=\frac{2 \pi b_{i}(\Delta f)_{i}}{8 P_{o} a_{i}\left(h+x_{i}\right)-\pi(\Delta f)_{i}}
$$

where, $a_{i}$ is the length of semi-major axis of the ellipse, $b_{i}$ is the length of semi-minor axis of the ellipse, $h$ is the height of finger and $x_{i}$ is the change in lateral sides of the chamber due to pressurization.

In order to find $x_{i}$, we have assumed that the initial volume of silicone is equal to the final volume of silicone around the joint after pressurization as used in [33].

$$
A_{\text {ini }} h=A_{\text {fin }}\left(h+x_{i}\right)
$$

$$
\begin{aligned}
& A_{i n i}=\pi a_{i}\left(b_{i}+t_{i}\right)-\pi a_{i} b_{i} \\
& A_{\text {fin }}=\pi a_{i}\left(b_{i}+t_{i}\right)-\pi a_{i}\left(b_{i}+\frac{(\Delta d)_{i}}{2}\right)
\end{aligned}
$$

where, $b_{i}$ is the length of semi-minor axis of the ellipse, $t_{i}$ is the thickness of soft silicone around the joint, $A_{i n i}$ is the initial area of silicone and $A_{f i n}$ is the final area of silicone around the joint after pressurization, respectively (see Fig.3.1(b)).

To determine the stiffness for each joint due to pressurization, we substituted Equation (3.3) into Equation (3.1).

$$
K_{i}+\Delta K_{i}=\frac{f_{o}}{d_{i}-\frac{2 \pi b_{i}(\Delta f)_{i}}{8 P_{o} a_{i}\left(h+x_{i}\right)-\pi(\Delta f)_{i}}}
$$

The results for vertical ellipse chamber shape in the Fig.3.2(a)(b) shows that increasing pressure at constant pulling length increases stiffness for both distal and proximal joints. 


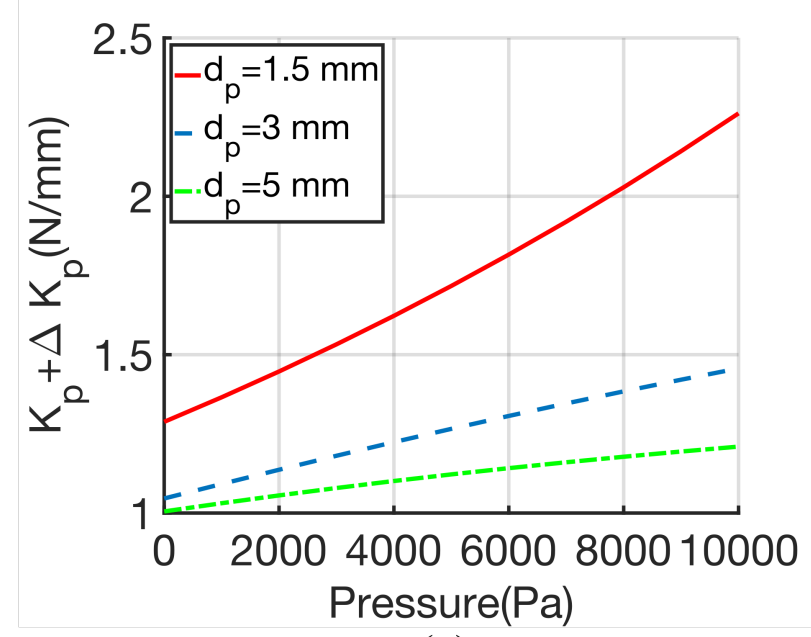

(a)

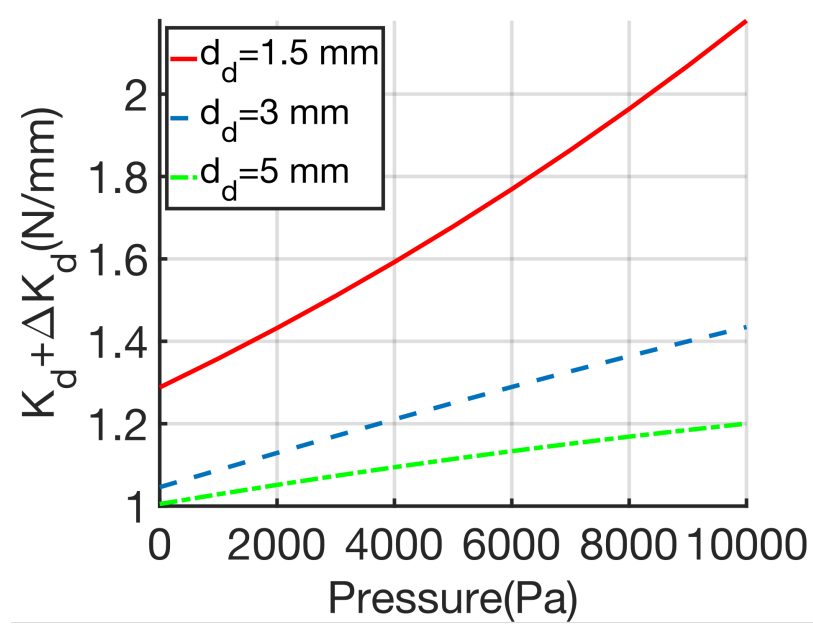

(b)

Figure 3.2: Increasing pressure stiffens the joints: (a) proximal joint (b) distal joint

\subsubsection{Finger Stiffness}

The increased joint stiffness leads to overall finger being stiffened. The constitutive relation for finger stiffness is similar to joint stiffness and can be written as:

$$
f_{o}=\left(K_{\text {finger }}+\Delta K_{\text {finger }}\right)\left(d_{\text {finger }}-\Delta d_{\text {finger }}\right)
$$

where, $K_{\text {finger }}$ is the stiffness constant of the finger and $\Delta K_{\text {finger }}$ is the additional stiffness constant due to pressurization, $d_{\text {finger }}$ is the cumulative deformation in linear spring of both joints 
and $\Delta d_{f i n g e r}$ is the total elongation in both air springs due to pressurization.

The stiffness of the finger due to pressurization is derived in a similar way as it was derived for the joint (see Equation (3.5)).

$$
\begin{gathered}
K_{\text {finger }}+\Delta K_{\text {finger }}=\frac{f_{o}}{d_{\text {finger }}-\Delta d_{\text {finger }}} \\
\Delta d_{\text {finger }}=(\Delta d)_{p}+(\Delta d)_{d}
\end{gathered}
$$

The results of the model are shown in Fig.6.1 and Fig.6.2 and they show that increasing pressure at all pulling lengths leads to stiffening of the finger.

\subsubsection{Firm Grasp}

A robot hand with stiffened fingers can firmly grasp the objects in dynamic situations because they have higher structural rigidity which help them constrain the object at multiple points (see Fig.3.3). Also, when the finger is pressurized keeping the tendon pulling length constant, this increases the joint angles due to the asymmetric design of the finger as inextensible tendons constraint the lower side (AA') and pressure expands the chamber (B'B and CC') (see Fig.3.3). This causes finger to apply higher normal force on the object which can decrease the slippage. This can also be shown by using a simplified model consisting of a spring $\left(K_{s}\right)$ attached at the fingertip. When the finger is pressurized, it exerts a push on the object which increases the deformation of the finger, $\Delta e$ thus resulting in higher normal force $\left(\Delta N=K_{s} \Delta e\right)$ on the object (see Fig.3.4).

In addition, we have modeled to show that increased normal force at a single point can reduce object slippage during manipulation. Initially, it is important to model frictional force between the soft tip and the rigid object. The Coulomb's model is not applicable when it comes to modeling contact forces between soft finger and rigid objects so a different formulation derived in [34] is considered for our case (see Fig.3.4).

$$
|f| \leq \alpha N+\pi \tau_{o} a^{2}
$$


where, $f$ is the friction force between finger tip and object, $\alpha$ is the rate of increase of shear strength, $\tau_{o}$ is the shear strength of surface force when load is zero [35], $a$ is radius of contact area and $N$ is the normal force.

Also, we derived the dynamics of the object to predict its behavior during manipulation [36].

$$
m_{o} \ddot{x_{2}}+f_{s}=-f
$$

where, $\ddot{x_{2}}$ is acceleration of the object, $f_{s}$ is the friction force when the object is stationary, $m_{o}$ is the mass of the object and $f$ is the friction force between soft finger tip and the object.

Equation (3.9) can be substituted into Equation (3.8) to derive a condition for acceleration at which the object can be manipulated without slipping from the fingers [34].

$$
\ddot{x_{2}} \leq \frac{N \alpha+\tau_{o} \pi a^{2}-f_{s}}{m_{o}}
$$

This condition validates the firm grip as increased normal force allows higher acceleration object manipulation keeping material parameters constant. 


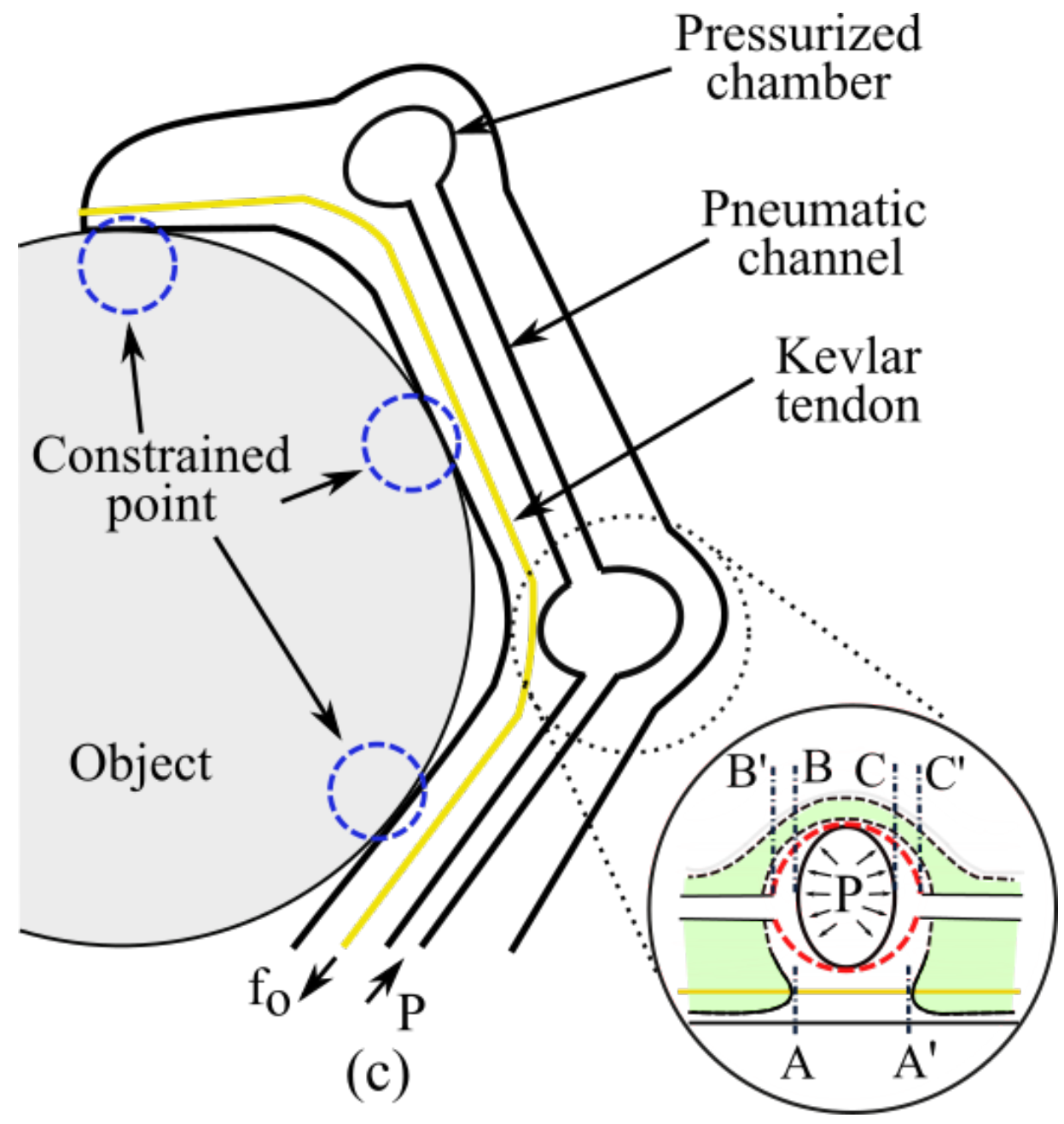

Figure 3.3: Soft composite finger with augmented structural rigidity to better constrain the object at multiple points. The inextensible tendon keeps AA' constant, whereas B'B and CC' increases with pressurization which exerts a push against the object. 

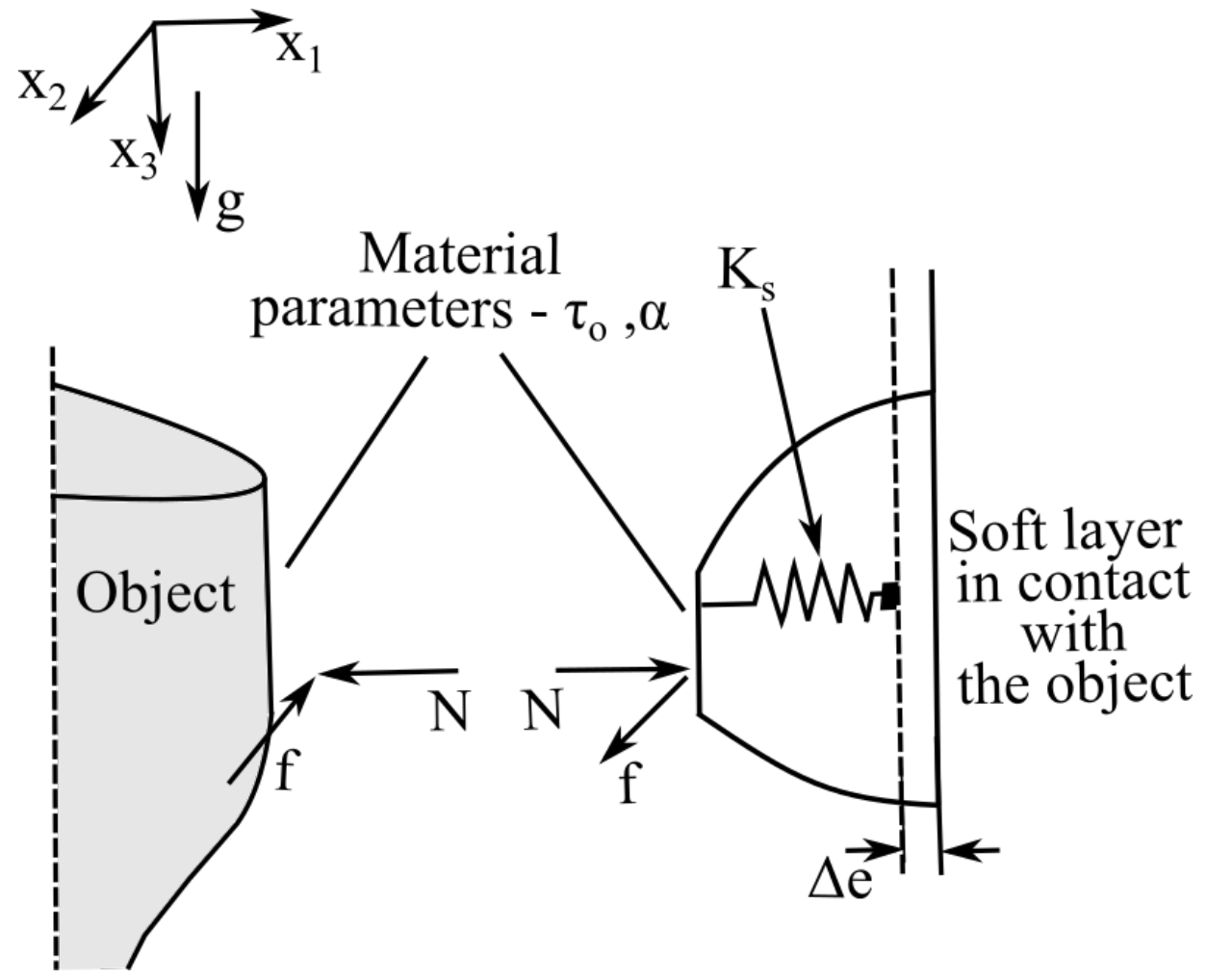

Figure 3.4: Modeling of normal force between soft finger tip and object. 


\section{FABRICATION}

The composite finger is fabricated from two translucent silicones: PDMS (Sylgard 184 - Dow Corning, MI, USA) and ECOFLEX 00-30 (Smooth-On Inc., PA, USA). These materials were chosen for their stability, strength, and ability to cohesively bond to one another. PDMS has a Shore A hardness of 50 and a tensile strength of $6.70 \mathrm{MPa}$ which makes it suitable to strengthen the overall structure of the finger. Meanwhile, ECOFLEX has lower Shore A hardness of 30 and tensile strength of 1.40 MPa which enhances the compliant nature of the finger that helps it to adapt to different kinds of objects in unstructured environments. In addition, it also augments the softness of the finger.

The composite finger consists of ECOFLEX encompassing a cured PDMS structure in 1:1 ECOFLEX-PDMS volumetric ratio. The design of the soft composite finger is inspired from morphology of the human finger. The PDMS structure consists of two thin arches connecting three distinct segments of different lengths, analogous to the proximal, middle, and distal phalanges of a human finger [37]. There are two joints similar to proximal and distal interphalangeal joints.

There are two channels for tendon actuation on the lateral side of the finger and one channel for pneumatic actuation in the center of the finger. The Kevlar thread (size 46, strength 14 Lbs, The Thread Exchange, NC, USA) is routed through tendon channels to allow tendon actuation. Whereas, a 2mm silicone tube (McMASTER-Carr, IL, USA) is cured in the pneumatic channel which connects two air chambers and continues to the proximal end of the finger to enable pneumatic actuation.

The PDMS and ECOFLEX are casted in 3D printed molds made of PLA filament $(1.75 \mathrm{~mm}$ Polylactic Acid, HATCHBOX 3D, CA, USA). Each mold has two unique knuckles that are used to fabricate both distal and proximal joints in the finger as shown in Fig.4.1(a). The PDMS and ECOFLEX cure around the knuckles, creating indentations in the structures when removed. Also, knuckles allow the cured silicones to be removed from the mold without damaging the silicone tubing. 
The finger fabrication process is outlined in Fig.4.1. The two metal rods are inserted in the base (proximal end) of the finger and extended to the tip to create channels through the structure for the tendons to be routed (see Fig.4.1(b)). Secondly, the silicone tube is sealed at one end using a silicone adhesive (Smooth-On Inc., PA, USA) to ensure that neither PDMS nor ECOFLEX goes into the tubing during the curing process. Then, the silicone tubing is inserted through the base and extended to the distal knuckle (see Fig.4.1(b)). A small piece of heat shrink (McMASTER-Carr, IL, USA) is also placed around the end of the tubing to avoid the silicone leakage. The knuckles are inserted into their designated spaces as shown in (see Fig.4.1(b)), securing the silicone tubing in place. The entire prepared mold is sprayed with an Ease Release Spray (Smooth-On Inc., PA, USA) to aid the removal process.

First, a PDMS casting is made. Sylgard 184 Elastomer Base and Curing Agent are mixed in 10 to 1 ratio. The mixture is left to settle for 15 minutes and then it is degassed thrice at -1 bar in a vacuum chamber to remove air bubbles from the mixture that could cause weakness in the PDMS structure. Then, the mixture is poured into the prepared mold and is left to cure at room temperature for 48 hours (see Fig.4.1(c)).

After the PDMS has cured, the needles and heat shrink are removed from the mold (see Fig.4.1(d)). The casting is carefully removed and inserted into the ECOFLEX mold [see Fig.4.1(e)]. The mold is prepared again, as previously explained (see Fig.4.1(f)). Then, ECOFLEX parts A and $\mathrm{B}$ are mixed in 1 to 1 ratio before degassing in the vacuum chamber. The mixture is poured into the mold which is left to cure at room temperature for 4 hours (see Fig.4.1 $(\mathrm{g})$ ).

The cured finger is removed from the mold. The silicone tubing is trimmed at the end to remove the silicone epoxy seal to pressurize the distal air chamber. A small piece of tubing is cut out of the proximal air chamber, allowing air to flow into the air chamber (see Fig.4.1(h)). The air chambers are sealed by placing the finger on top of a shallow tray filled with PDMS to create an airtight seal over the chambers as shown in (see Fig.4.1(i)).

The Kevlar thread is routed through the parallel tendon channels such that the free ends of the thread exit at the base of the finger. The finger bends when tension is applied to Kevlar threads 
causing the composite finger to bend in a discrete manner. The end of the silicone tubing is connected to pneumatic system to stiffen the finger (see Fig.4.1(j)). The pneumatic system consists of $3 \mathrm{~V}$ and $6 \mathrm{~V}$ mini air pump motors (Uxcell, New Territories, Hong Kong) used to pressurize the finger, solenoid valve (Uxcell, New Territories, Hong Kong) to control the flow of air and a microcontroller (Trinket 5V, Adafruit, New York, NY, USA) board to control the timing of air pump motor and valve.

Moreover, in order to see the effect of chamber shape on the performance of finger, different shapes were fabricated in a similar way as explained earlier. Vertical ellipse (see Fig.4.2(a)) was chosen on the basis of embedding a joint which can enhance local stiffening capability and promote discrete bending. As the rectangular cross-sectional area determines the local stiffening at the joint, so circle (see Fig.4.2(b)) was derived from vertical ellipse with similar rectangular cross-sectional area to see its effect on the performance of the finger. As volume of air going into these chambers play significant role in stiffening so small circle (see Fig.4.2(c)) was adopted as it can store similar volume of air as vertical ellipse. Horizontal ellipse (see Fig.4.2(d)) is exactly similar to vertical ellipse except the orientation of the ellipse. 


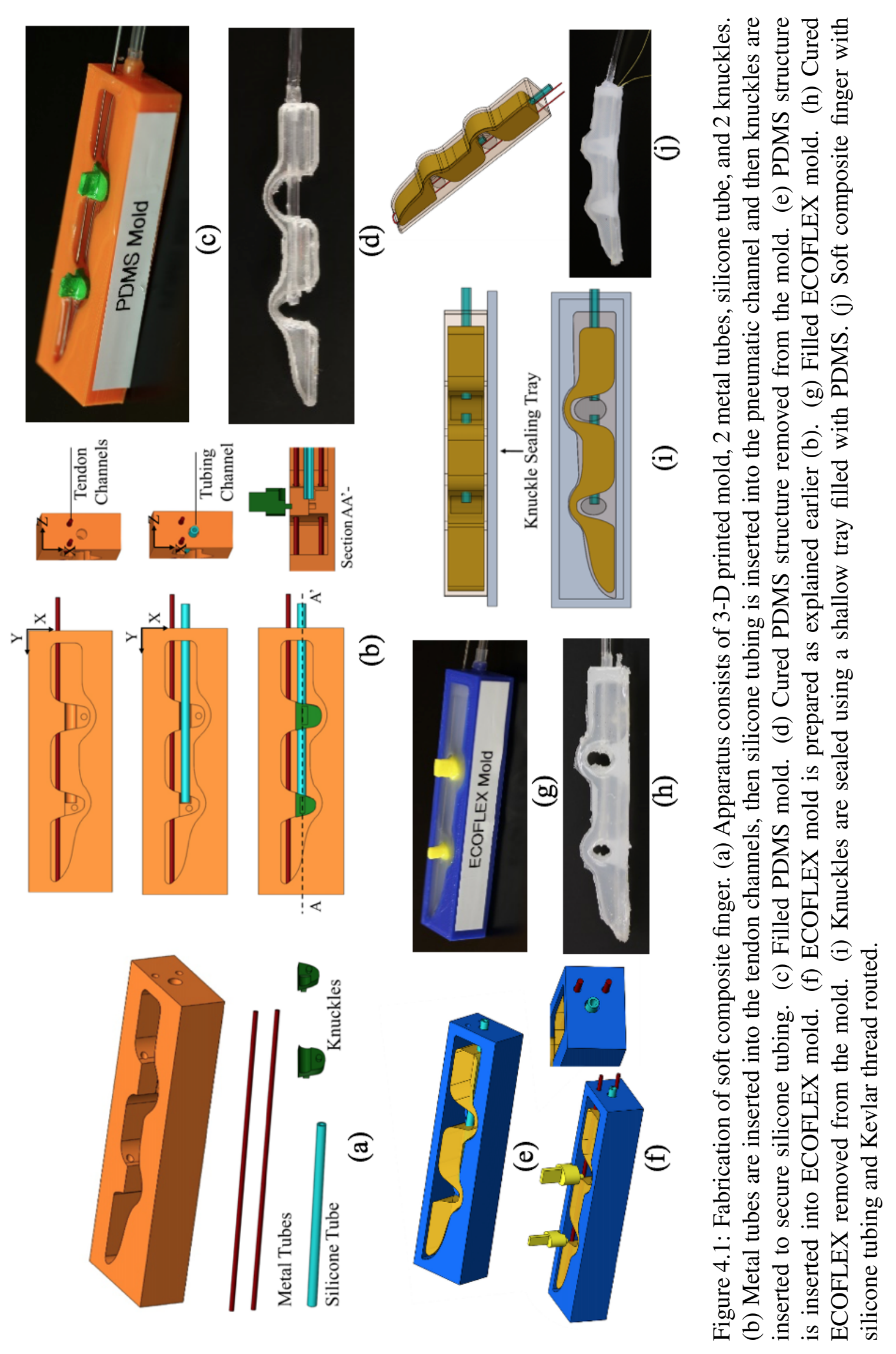


(a)

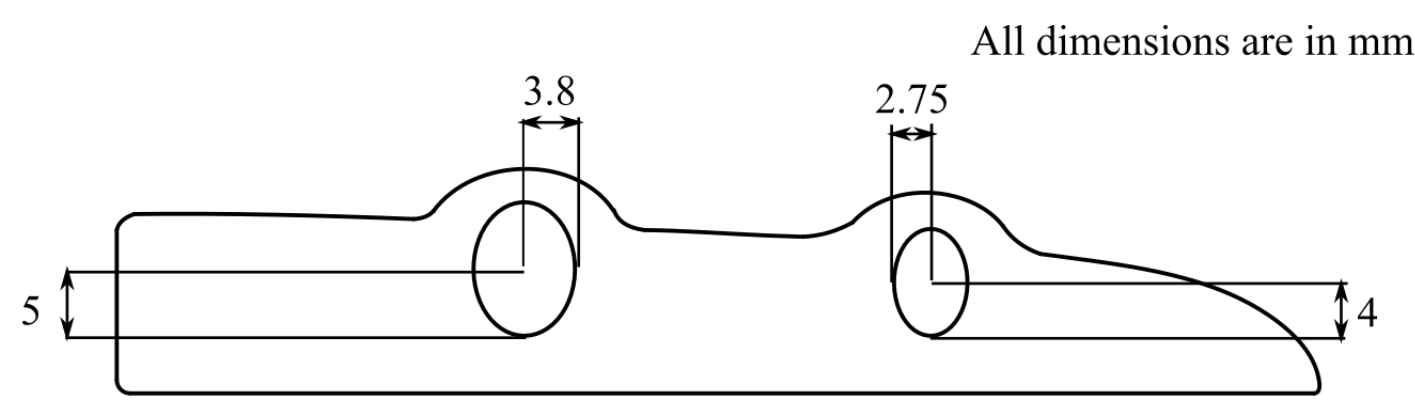

(b)

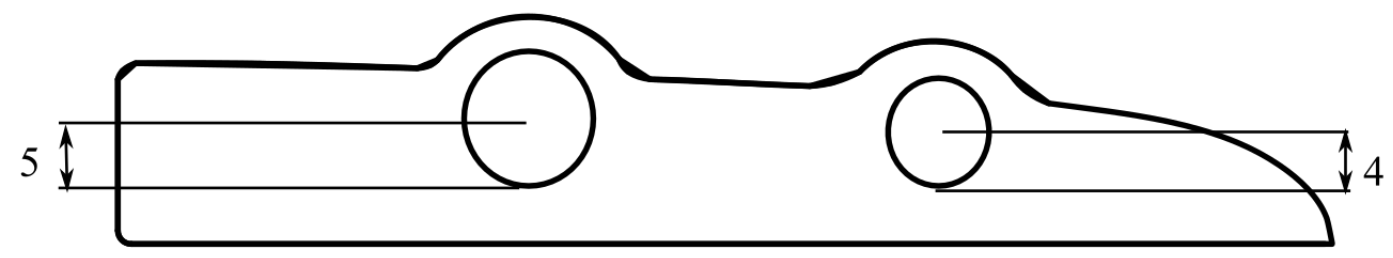

(c)

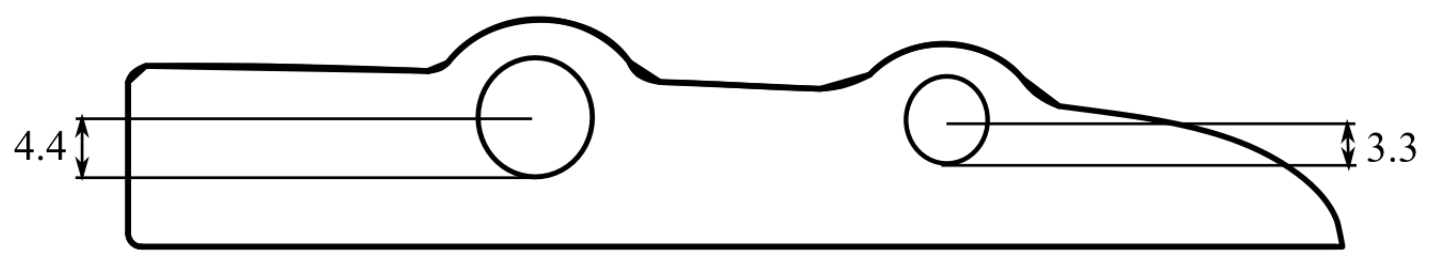

(d)

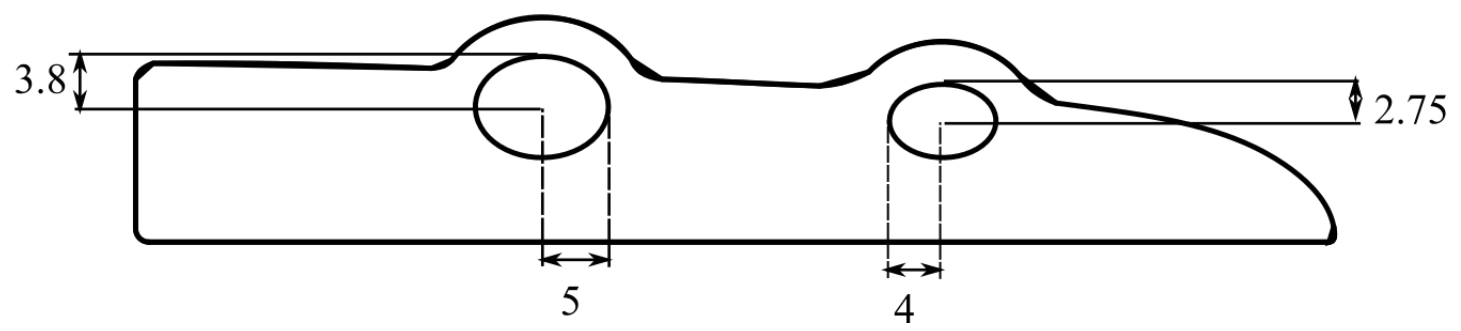

Figure 4.2: Different finger designs. (a) Vertical Ellipse (b) Circle (c) Small Circle (d) Horizontal Ellipse. 


\section{EXPERIMENTS}

\subsubsection{Stiffness Experiments}

The first set of experiments were conducted to investigate the effect of increasing pressure on overall stiffness of the finger. The experiments consisted of pulling the tendons for different displacements ( $3 \mathrm{~mm}, 7 \mathrm{~mm}, 12 \mathrm{~mm}$ ) and measuring the resulting force needed to bend the finger using a six DOF force sensor (Nano 17, ATI Industrial Automation, NC, USA) as shown in Fig.5.1(a). The sensor was attached to a 3-D printed part mounted on the motorized linear platform. The data from the force sensor was recorded at $1 \mathrm{~Hz}$ using a DAQ card (USB-6210, National Instruments, TX, USA). The same procedure was also conducted at higher pressures (6600 $\mathrm{Pa}$ and $10400 \mathrm{~Pa}$ ) to get stiffness values. The raw force displacement data was used to calculate overall stiffness of the finger. This experiment was also repeated for different chamber shapes to see the effect of pressurization on them.

\subsubsection{Joint Angle Experiments}

As the soft composite finger shows discrete bending behavior when Kevlar tendons are pulled so the second set of experiments were performed to characterize this behavior in terms of joint angle (see Fig.5.1(b)). The finger was marked before the experiment to evaluate the joint angles as shown in Fig.5.1(b). The first line was drawn parallel to the fixture, following the second line connecting the center of both joints and parallel to middle segment. The third line connects the center of distal joint to the finger tip. The Kevlar tendons routed in the finger were attached to 3-D printed part fixed to a motorized linear mechanism which was programmed to displace for $3 \mathrm{~mm}, 7$ $\mathrm{mm}$ and $12 \mathrm{~mm}$ at a constant velocity. A high-definition camera (EOS Rebel SL1, Canon Inc., NY, USA), was used to capture photos of the soft composite finger at all displacements. This routine was repeated at higher pressures (6600 $\mathrm{Pa}$ and $10400 \mathrm{~Pa}$ ) to see the effect of pressurizing on joint angles or discrete behavior.

This experiment was also done for different chamber shapes to see their effect on bending of 


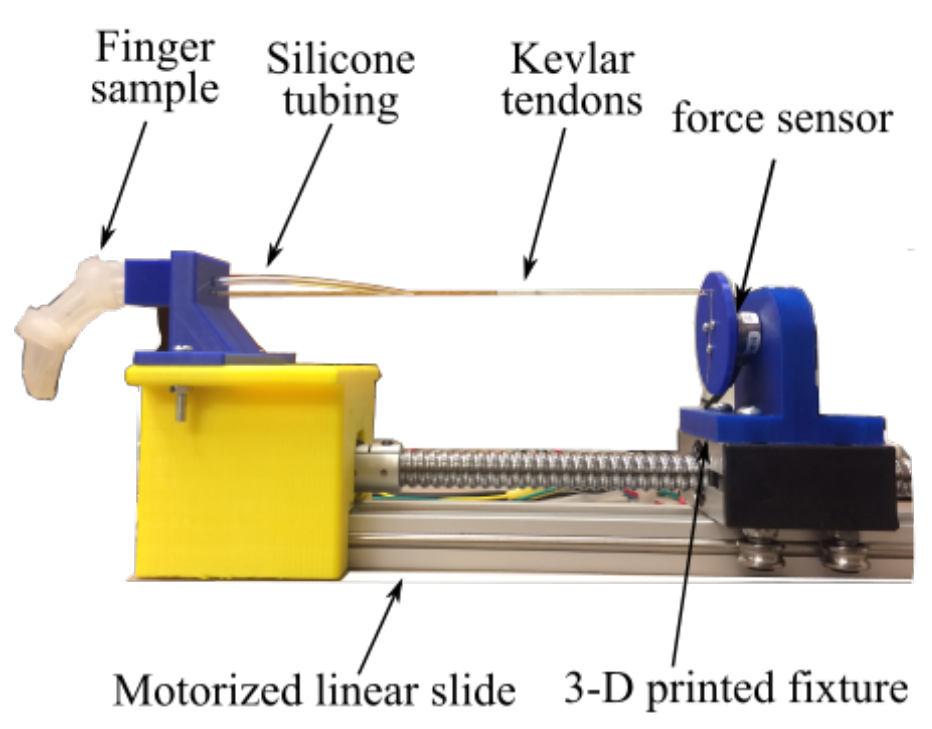

(a)
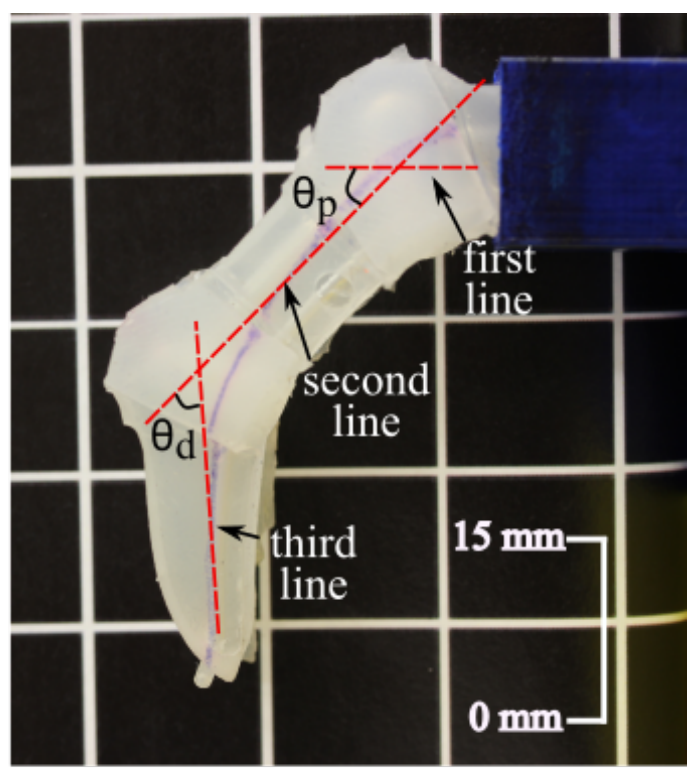

(b)

Figure 5.1: Experimental setups for soft composite finger. (a) Experimental setup for stiffness tests using force sensor and motorized linear module. (b) Soft composite finger showing discrete bending behavior.

the finger. Finally, all the captured photos were analyzed using a raster graphics editor software to calculate the joint angle for both joints of the finger. This set of experiments were repeated after substantial time to avoid the interference of viscoelastic nature of silicones as it significantly effects the following set of trials.

\subsubsection{Firm grasp Experiments}

To validate that stiffening joints leads to firm grasp over the object, we have designed an experiment consisting of a single link robotic arm attached to a gripper made up of three soft fingers with vertical ellipse chamber (see Fig.6.7(b)). In this experiment, the robotic arm carrying a wine glass was rotated about its axis at increasing amplitudes of acceleration until the wine glass slips out of the gripper. This experiment was conducted for two cases (No pneumatic actuation and With pneumatic actuation). In addition, this experiment was repeated with weights ( $140 \mathrm{~g})$ to substantiate the effect of mass on stiffening further (see Equation (3.10)). 


\section{RESULTS AND DISCUSSION}

We tested different chamber shapes as described earlier to demonstrate the stiffening effect due to pressure. Both experimental results and model predictions indicate that an increased pressure stiffens the finger (see Fig.6.1 and Fig.6.2). The results also validate our modeling approach using a single air-spring along the minor axis of an elliptical chamber, aligned with the finger axis (see Fig.3.1). On the other hand, circular chambers (see Fig.6.1(b) and Fig.6.2(a)) may need multiple springs, and the horizontal ellipse (see Fig.6.2(b)) should be modeled using a dominant spring perpendicular to the finger axis, both of which conflict with the modeling assumption. As a result, the model of the vertical ellipse better matches the experiment (see Fig.6.1(a)), in particular, at low tendon length and low pressure that may satisfy the simple linear-spring model of the composite structure made from non-linear silicone materials. Moreover, the directional effect of the dominant air-spring is also confirmed in the bending angle test.

From the results plotted, it is possible to notice that all chamber designs show non-zero stiffness, $K_{\text {finger }}$ at zero pressure. Secondly, it is evident that increasing pulling length at constant pressure decreases overall stiffness of the finger. At higher pulling lengths, both distal and proximal chambers deform significantly due to the bending (cross-sectional area loss) of the finger so it becomes difficult for pneumatics to stiffen the finger. Although, all chamber shapes demonstrate increase in stiffness when pressure is increased, small circle shows highest stiffness as compared to other chamber shapes (see Fig.6.2(a)). The small circle has a similar volume to vertical ellipse but it has a higher stiffness probably because it has a smaller semi-major axis, $a_{i}$ (see Equation $(3.5))$.

Despite the fact that both vertical ellipse and circle have similar rectangular cross-sectional area, the stiffness of circle is less than that of vertical ellipse (see Fig.6.1(b)). According to Equation (3.2), vertical ellipse can store less volume of air which leads to higher force due to pressurization, thus higher stiffness. The results also show that changing the orientation of vertical ellipse to horizontal ellipse decreases the overall stiffness of the finger (see Fig.6.2(b)) probably due to the 
smaller cross sectional area as compared to vertical ellipse which decreases its stiffening capability (see Equation (3.2)).

In order to characterize the discrete bending of the finger, we performed joint angle experiments. It is evident from the results that increasing the pulling length increases both joint angles (distal and proximal) at fixed pressure values which leads to an observation that the finger is able to bend in a discrete manner (see Fig.6.3, Fig.6.4, Fig.6.5, Fig.6.6). The magnitude of proximal and distal angles seem to depend on the length of the longitudinal axis of the chamber. As the length of the longitudinal axis increases, the proximal and distal angle decreases with small circle having smallest and horizontal ellipse having the largest longitudinal axis. The results also show a similar behavior for vertical ellipse, circle and small circle as increasing the pressure at constant pulling length increases the joint angle. These increased joint angles due to high pressure results in an increased curvature at the joints which generates a push against the object leading to the firm grasp. Whereas, increasing the pressure at constant pulling length decreases joint angle for the horizontal ellipse (see Fig.6.6(a)(b)). In the case of horizontal ellipse, the dominant air spring should be perpendicular to the finger axis, which straightens the finger when pressurized, thus decreasing joint angles at higher pressures. In addition, distal joint angle is greater than the proximal joint angle in the vertical ellipse which shows that the finger is more compliant at the distal joint and it is in line with the behavior shown by prior soft robots [20].

Although, we have shown through model and experiments that pressurization stiffens the finger, there is a need to prove experimentally that the stiffening leads to firm grasp over the object. The ability to achieve firm grasp will allow manipulation of heavier objects even at higher accelerations, according to Equation (3.10). In order to verify the firm grasp of soft composite finger, we performed a wine glass manipulation experiment. Table 6.1 summarizes the results of this experiment. Comparing both cases, it can be noticed that without the stiffening, the gripper is not able to hold object with more mass (wine glass with weights) at higher acceleration. The stiffened fingers are structurally rigid, which constrain the object better and increase normal force on the object, allowing higher object acceleration as supported analytically by Equation (3.10). In addition, soft 
composite fingers can grasp objects of varying size, shape and also in different orientation (see Fig.6.7).

Table 6.1: Summarized results of firm grasp experiment on a Wine glass for two cases (Empty and with weights)

\begin{tabular}{|l||l|l|l|}
\hline \multicolumn{4}{|c|}{ Wine Glass(Empty) - Acceleration $\left(\mathrm{m} / \mathrm{s}^{2}\right)$} \\
\hline Pressure & 17 & 19 & 21 \\
\hline No pressure & Pass & Pass & Fail \\
\hline Pressure $(10400 \mathrm{~Pa})$ & Pass & Pass & Pass \\
\hline
\end{tabular}

\begin{tabular}{|l|l|l|l|}
\hline \multicolumn{4}{|c|}{ Wine Glass(with weights) - Acceleration $\left(\mathrm{m} / \mathrm{s}^{2}\right)$} \\
\hline Pressure & 17 & 19 & 21 \\
\hline No pressure & Pass & Fail & Fail \\
\hline Pressure $(10400 \mathrm{~Pa})$ & Pass & Pass & Fail \\
\hline
\end{tabular}




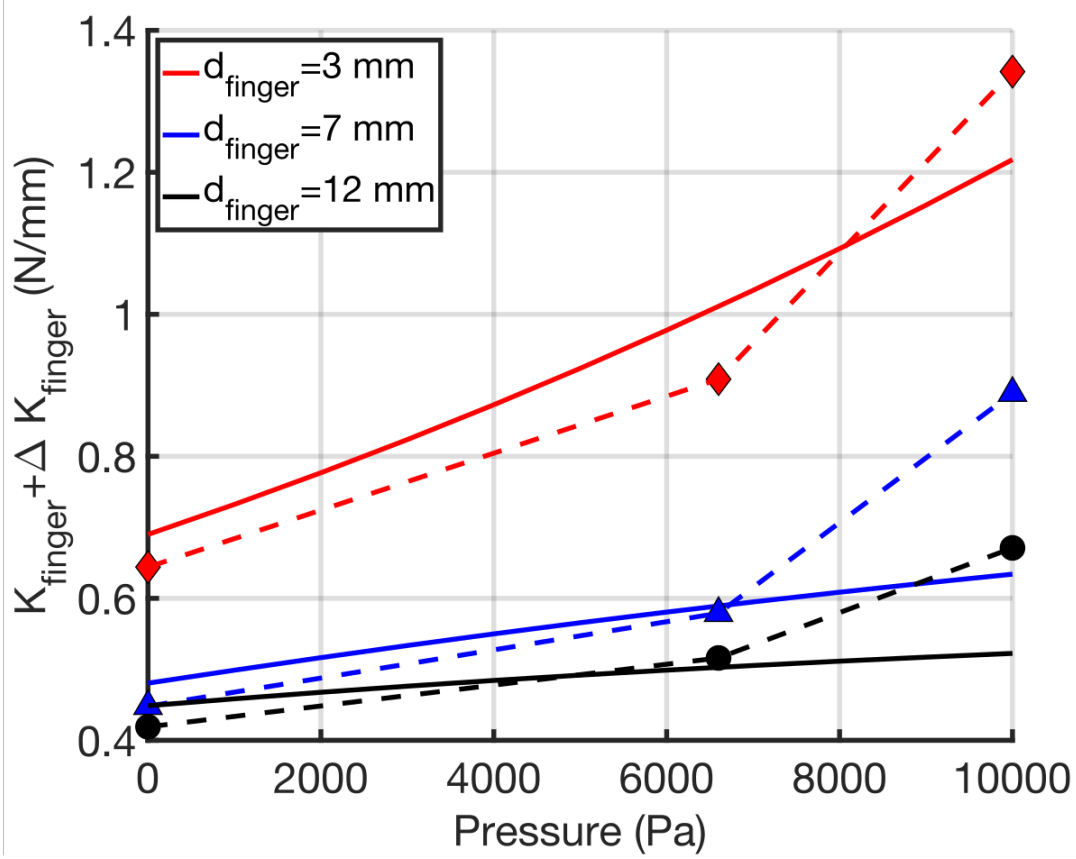

(a)

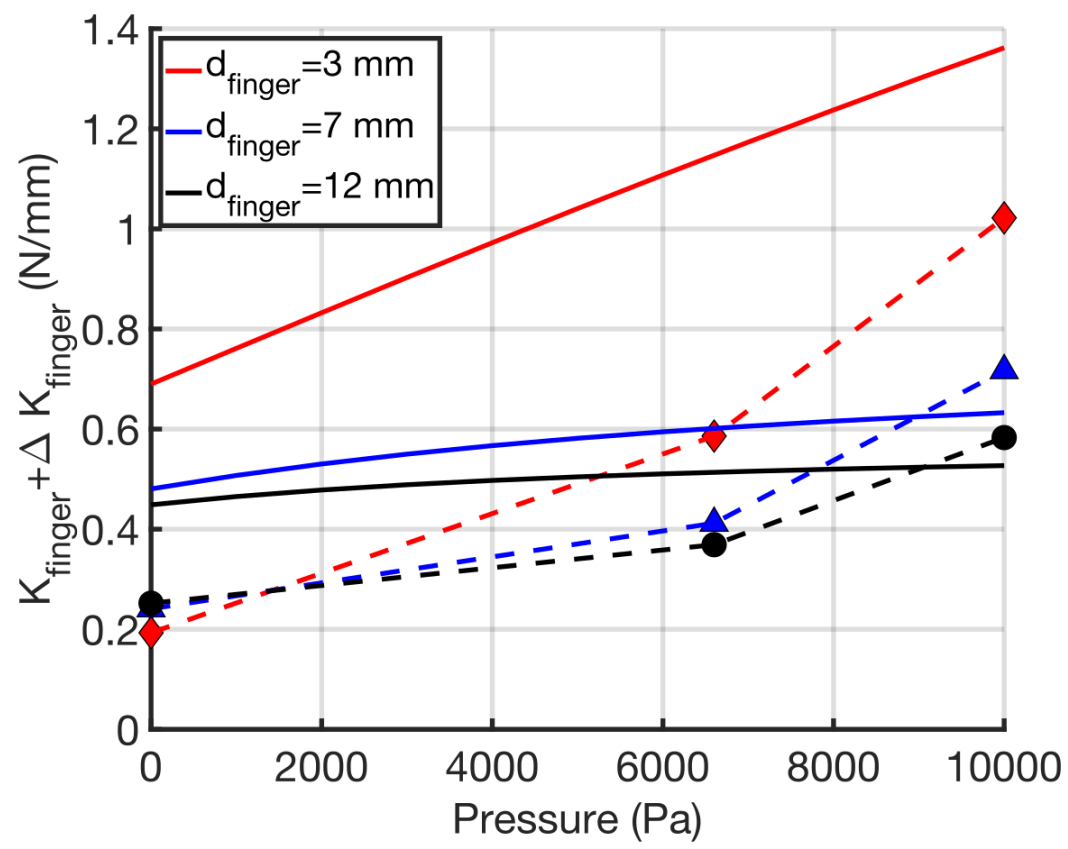

(b)

Figure 6.1: Both experimental results (connected by dashed lines) and model predictions (connected by solid lines) show that soft composite finger with different chamber shapes stiffens when the chambers are pressurized at constant pulling lengths of $3 \mathrm{~mm}, 7 \mathrm{~mm}, 12 \mathrm{~mm}$. (a) Vertical ellipse (b) Circle. As expected, the model of the vertical ellipse satisfying all underlying assumptions better predicts the experimental results. 


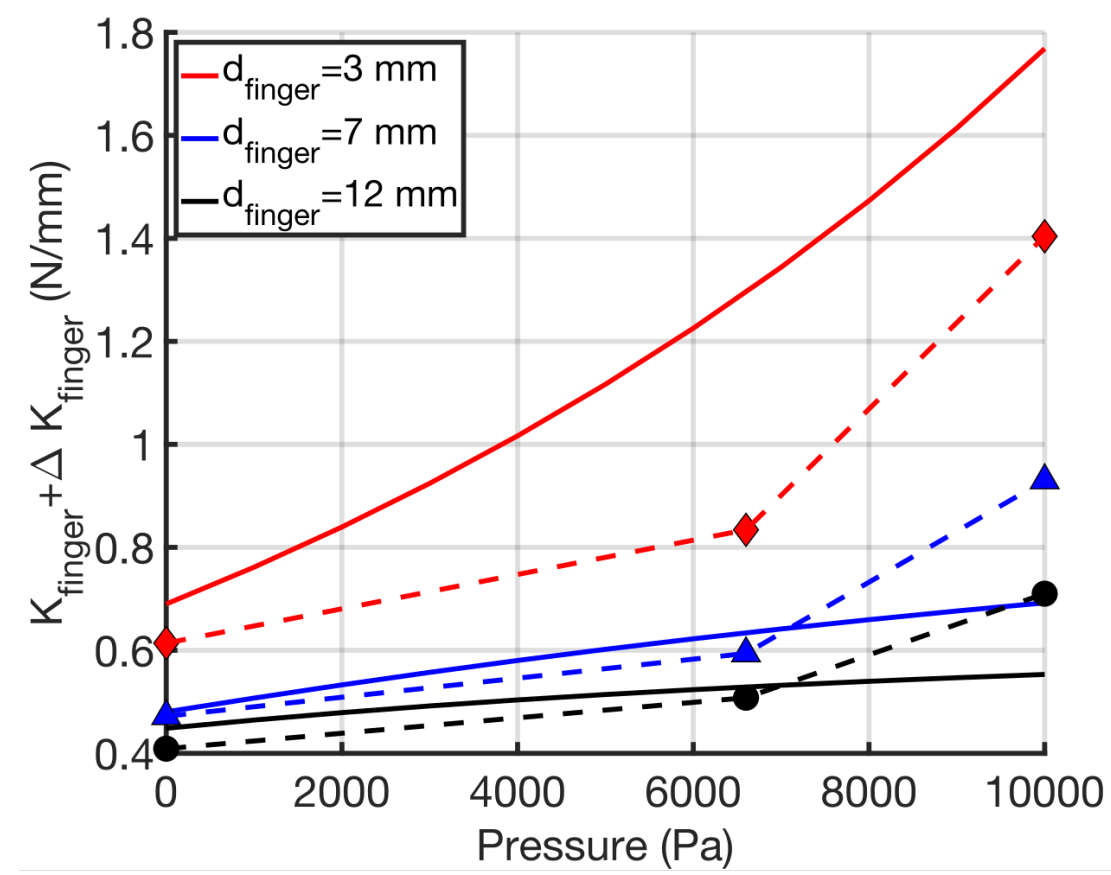

(a)

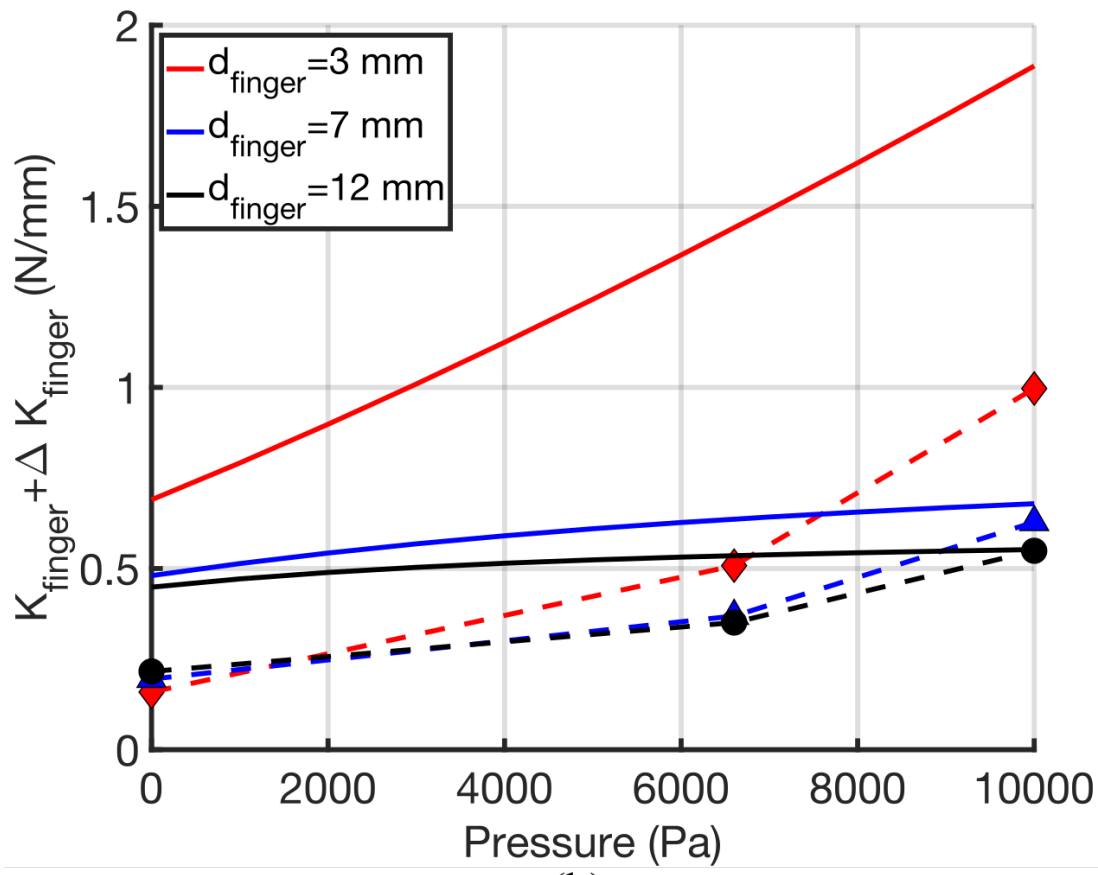

(b)

Figure 6.2: Both experimental results (connected by dashed lines) and model predictions (connected by solid lines) show that soft composite finger with different chamber shapes stiffens when the chambers are pressurized at constant pulling lengths of $3 \mathrm{~mm}, 7 \mathrm{~mm}, 12 \mathrm{~mm}$. (a) Small circle (b) Horizontal ellipse 


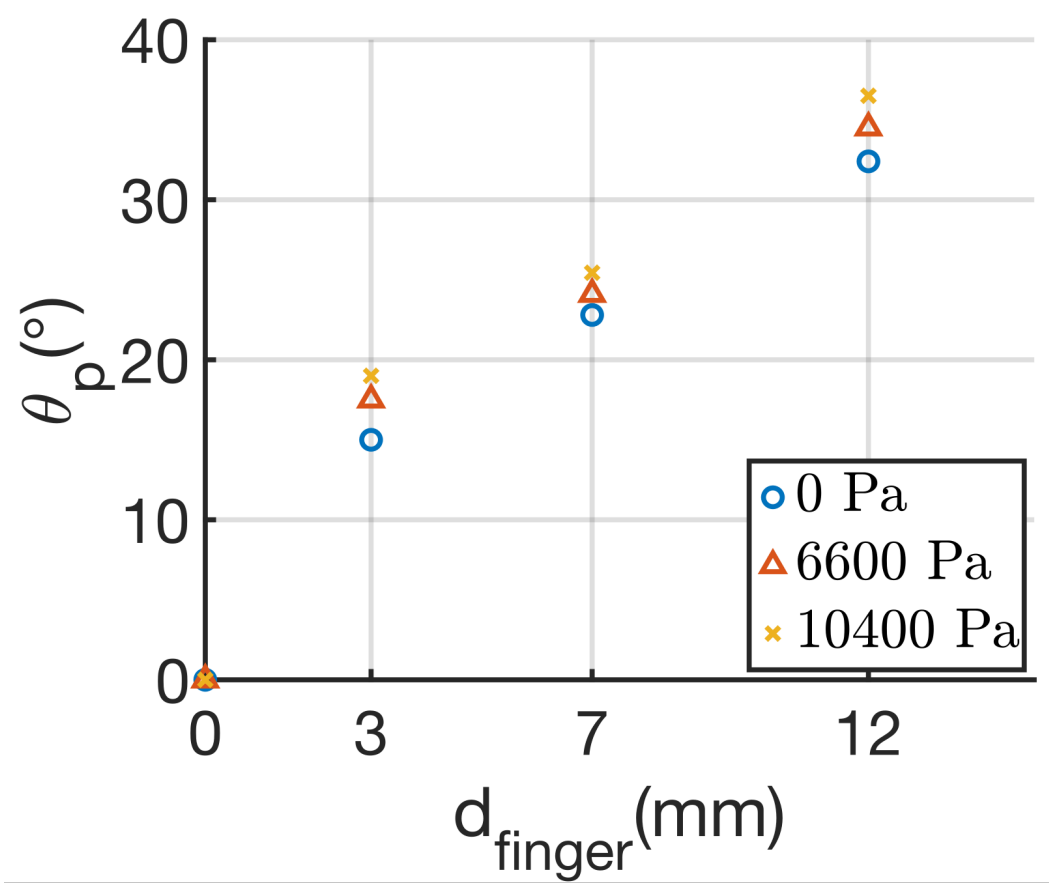

(a)

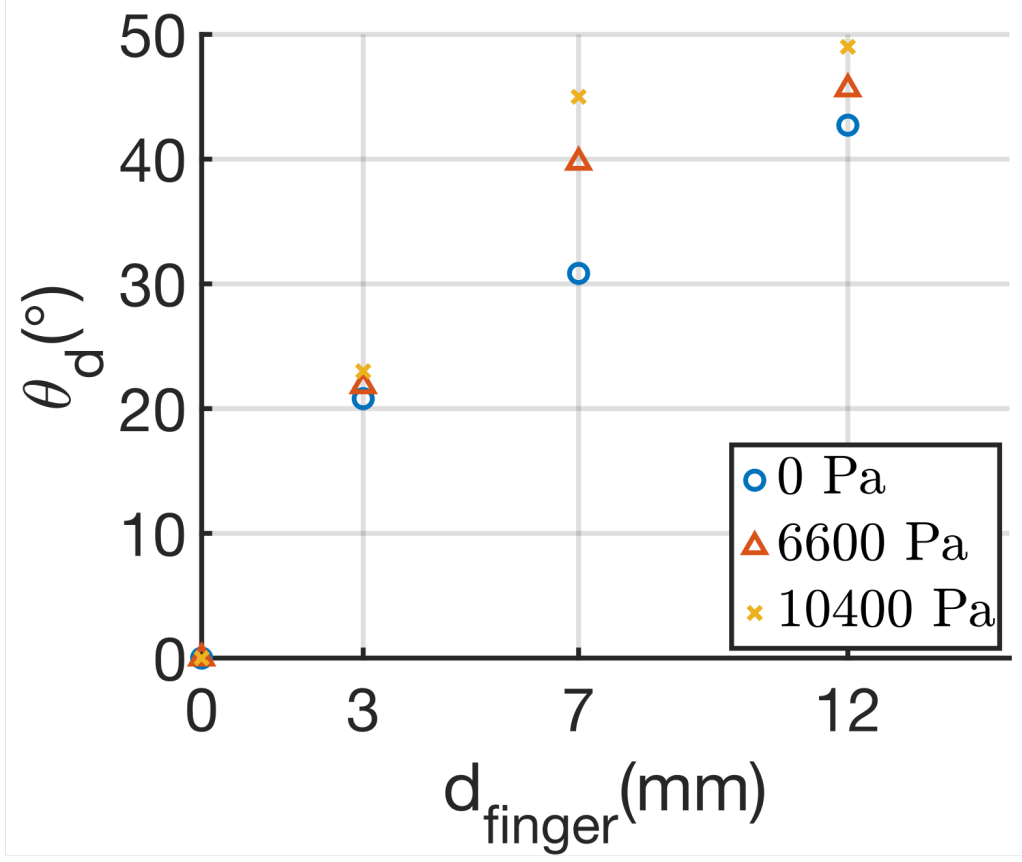

(b)

Figure 6.3: The soft composite finger shows discrete bending behavior when the tendons are pulled at constant pressures of $0 \mathrm{~Pa}, 6600 \mathrm{~Pa}, 10400 \mathrm{~Pa}$. (a) Vertical ellipse (proximal) (b) Vertical ellipse (distal). 


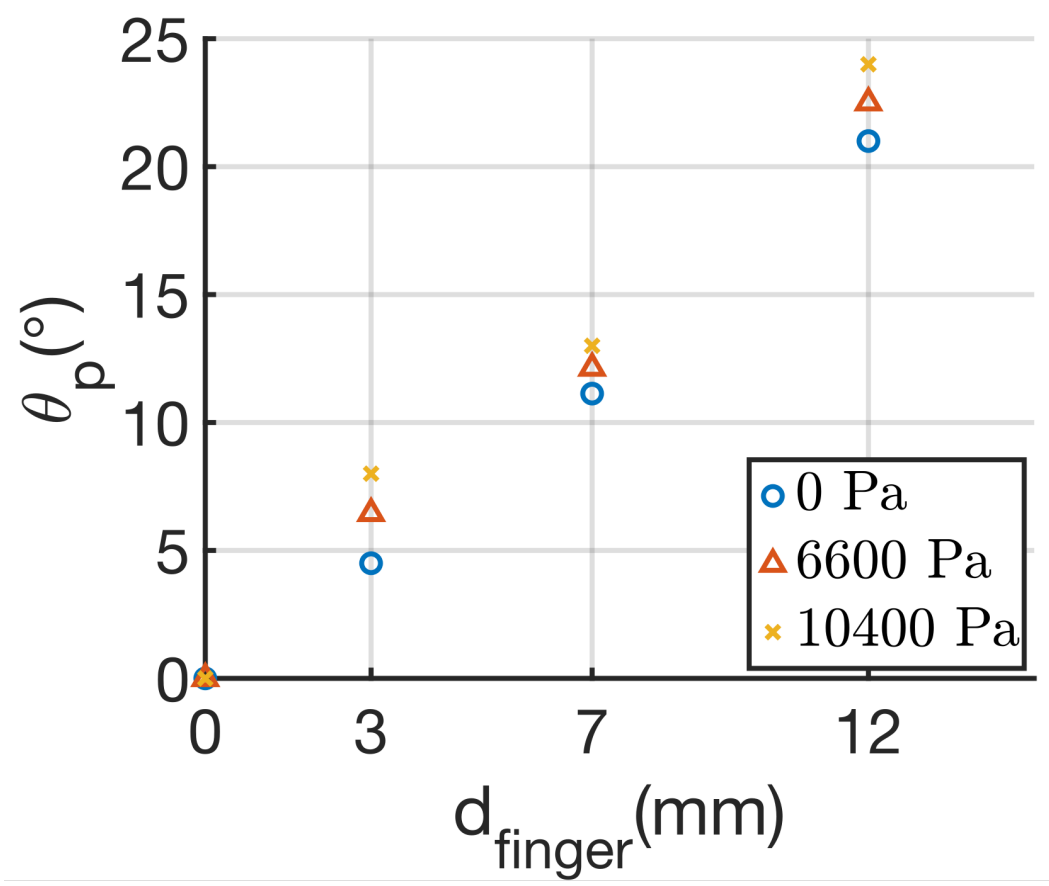

(a)

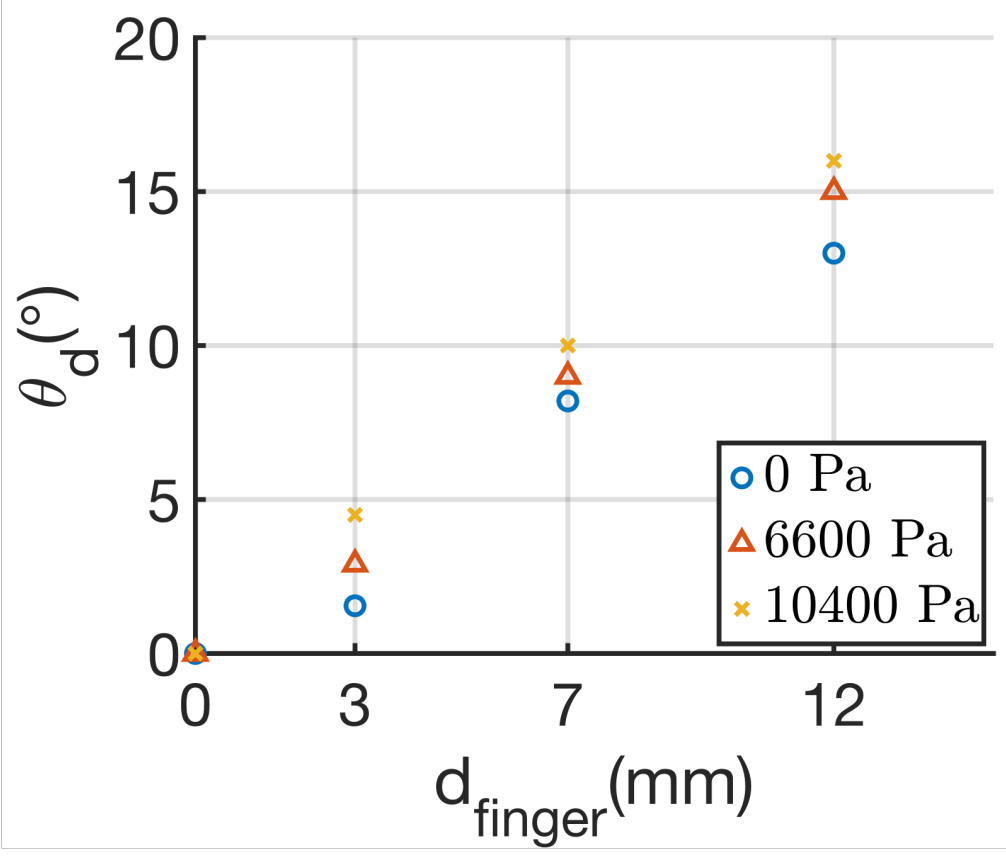

(b)

Figure 6.4: The soft composite finger shows discrete bending behavior when the tendons are pulled at constant pressures of $0 \mathrm{~Pa}, 6600 \mathrm{~Pa}, 10400 \mathrm{~Pa}$. (a) Circle (proximal) (b) Circle (distal). 


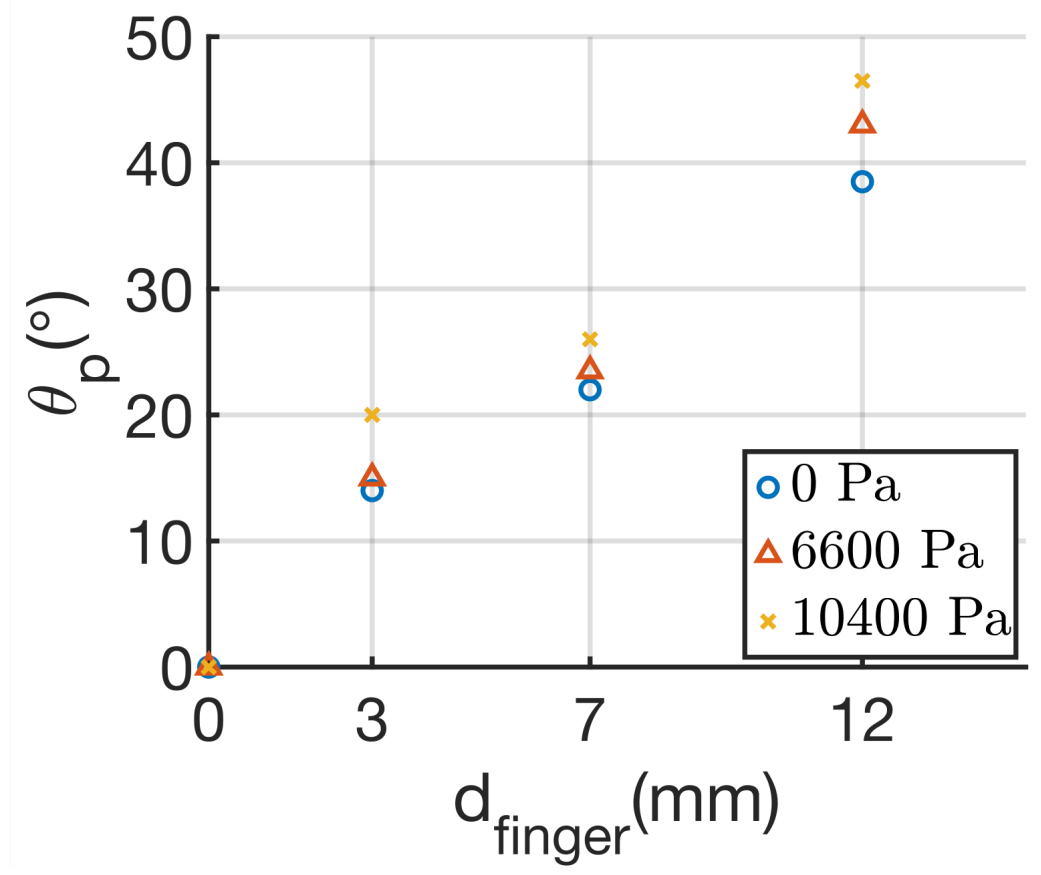

(a)

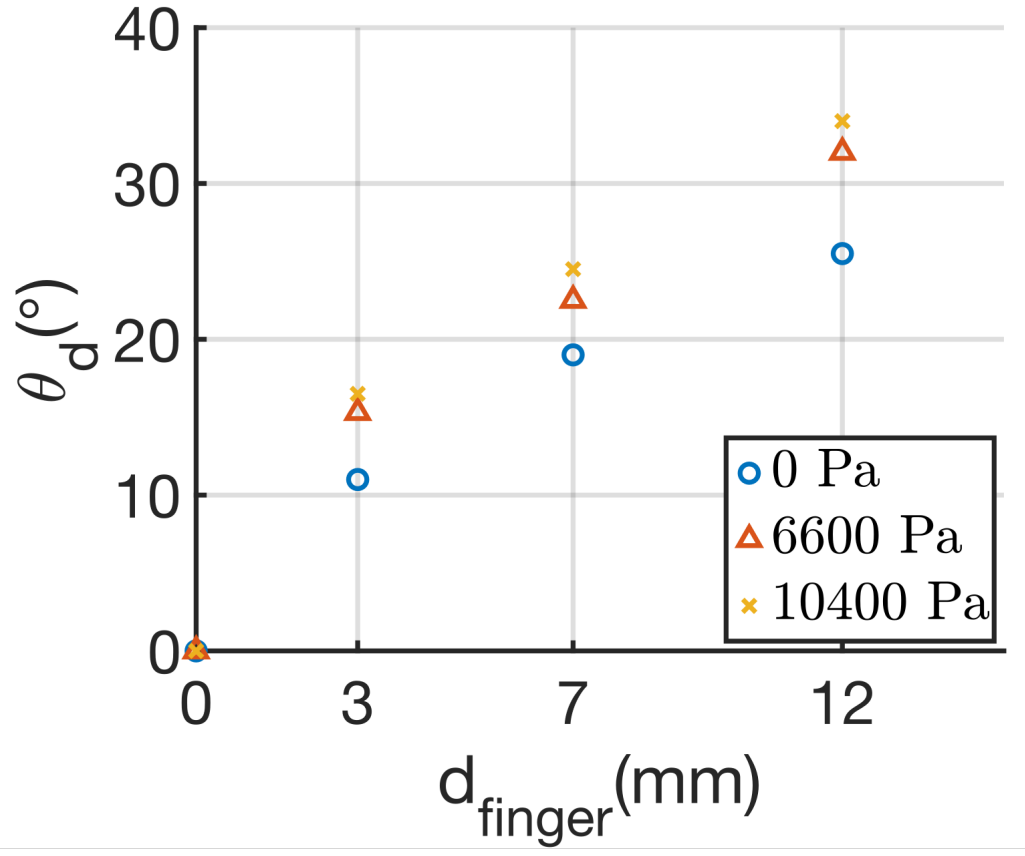

(b)

Figure 6.5: The soft composite finger shows discrete bending behavior when the tendons are pulled at constant pressures of 0Pa, 6600Pa, 10400Pa. (a) Smallcircle (proximal) (b) Smallcircle (distal). 


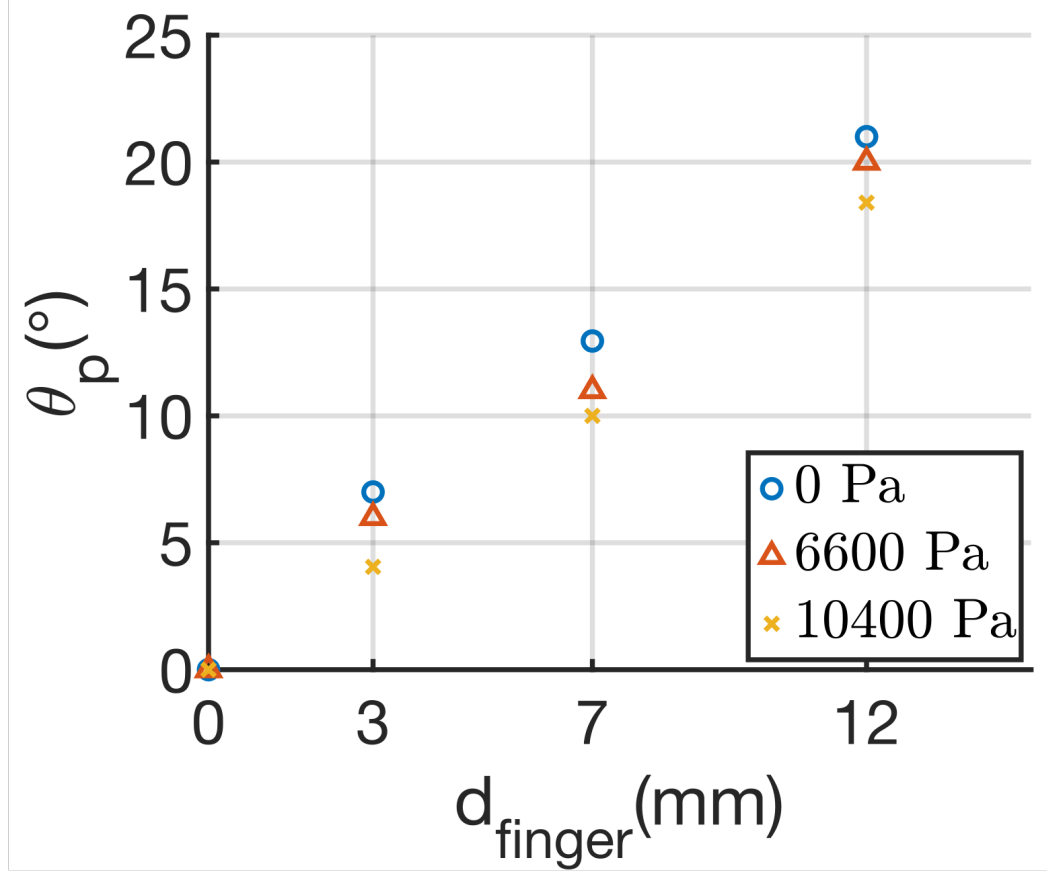

(a)

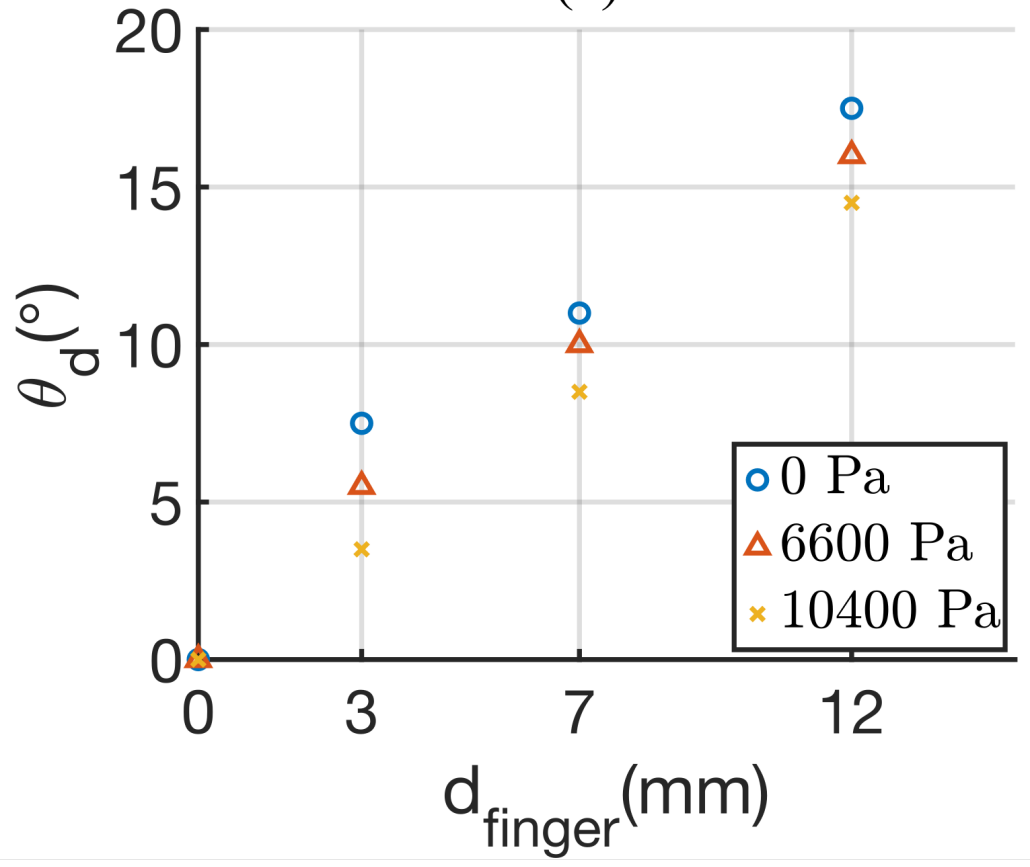

(b)

Figure 6.6: The soft composite finger shows discrete bending behavior when the tendons are pulled at constant pressures of $0 \mathrm{~Pa}, 6600 \mathrm{~Pa}, 10400 \mathrm{~Pa}$. (a) Horizontal ellipse (proximal) (b) Horizontal ellipse (distal). 


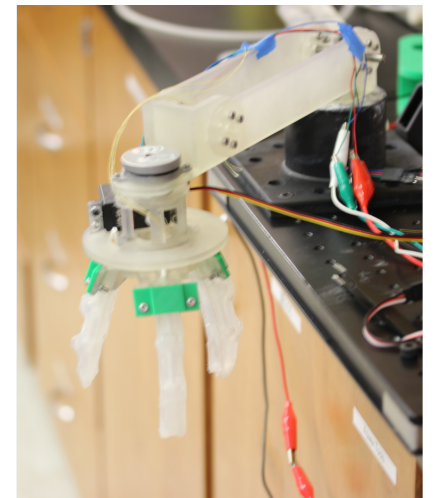

(a)

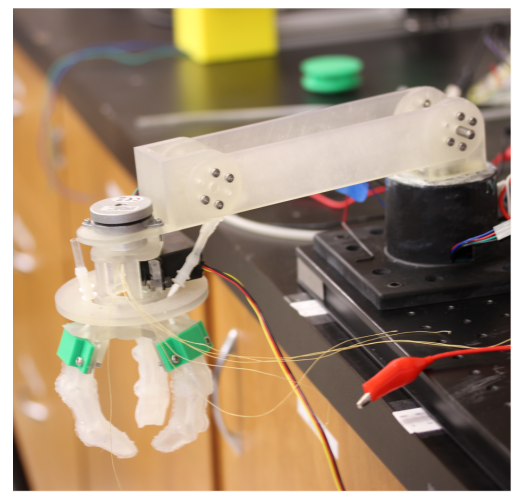

(e)

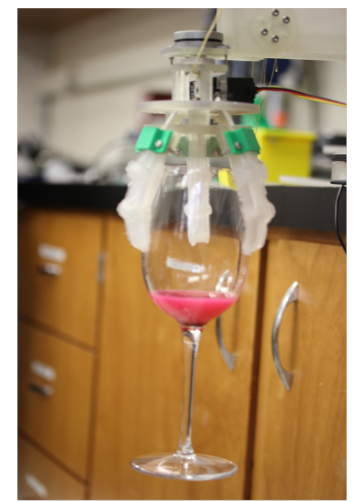

(b)

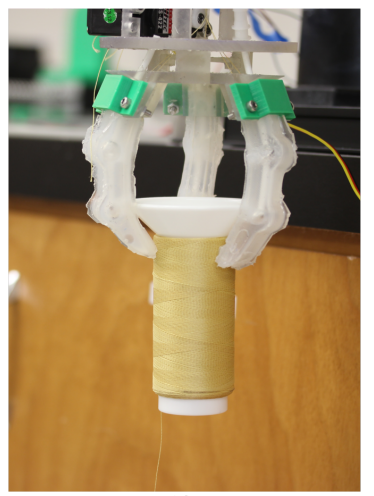

(f)

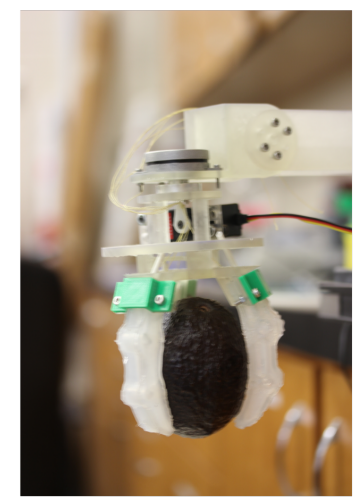

(c)

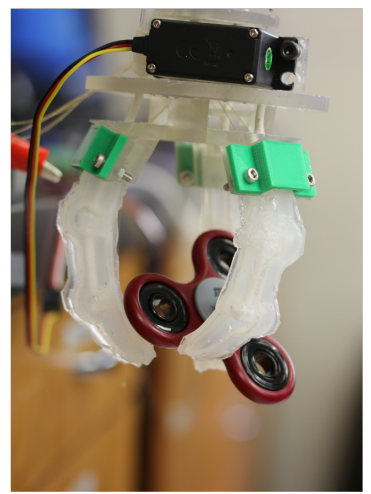

(g)

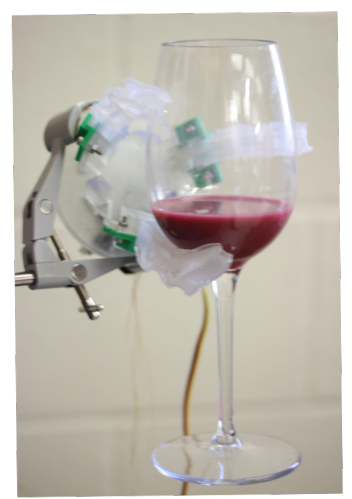

(d)

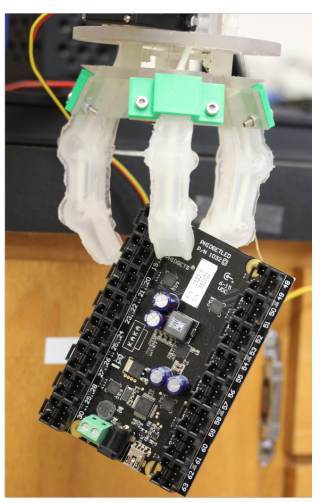

(h)

Figure 6.7: The designed gripper can grasp various size(diameter from $20 \mathrm{~mm}$ to $100 \mathrm{~mm}$ ) and shape objects such as circular, rectangular, oval, cylindrical, etc. at various orientations. (a) Robotic arm with gripper (b) Wine glass (c) Avocado (d) Wine glass grasped in a different orientation (e) Robotic arm with gripper actuated (f) Kevlar Spool (g) Fidget Spinner (h) Microcontroller board. 


\section{CONCLUSION AND FUTURE WORKS}

In this thesis, we have proposed a design of a soft composite finger with two embedded soft joints inspired from a human finger. Hybrid actuation combining both tendon and pneumatic actuation is implemented in the finger to bend it in a discrete behavior and stiffen the joints respectively. The model prediction and experimental results suggest that anthropomorphic soft composite fingers possess stiffening capability and can discretely bend with varying curvature along its length. Also, the key factors such as cross-sectional area and longitudinal axis of the joints effect the performance of finger in terms of stiffening capability and discrete bending behavior respectively. The stiffened fingers constrains the object better and apply increased normal force which enhances firm grasp over the object in dynamic situations.

In future works, viscoelasticity of the materials should be accounted in the stiffness model to better predict the dynamic performance of the finger at higher pulling lengths and pressure. Also, further research will focus on optimizing the design of embedded joints in the finger to get higher stiffness at higher pulling length which will enhance firm grasp behavior of the finger. 


\section{REFERENCES}

[1] U. Çulha and F. Iida, "Enhancement of finger motion range with compliant anthropomorphic joint design,” Bioinspiration \& biomimetics, vol. 11, no. 2, p. 026001, 2016.

[2] A. Kapandji, M. J. Kandel, and I. Kapandji, Physiology of the Joints: Lower Limb: Volume 2. Churchill Livingstone, 1988.

[3] A. Kochan, "Shadow delivers first hand," Industrial robot: an international journal, vol. 32, no. 1 , pp. $15-16,2005$.

[4] A. D. Deshpande, Z. Xu, M. J. V. Weghe, B. H. Brown, J. Ko, L. Y. Chang, D. D. Wilkinson, S. M. Bidic, and Y. Matsuoka, "Mechanisms of the anatomically correct testbed hand," IEEE/ASME Transactions on Mechatronics, vol. 18, no. 1, pp. 238-250, 2013.

[5] F. Ilievski, A. D. Mazzeo, R. F. Shepherd, X. Chen, and G. M. Whitesides, "Soft robotics for chemists," Angewandte Chemie, vol. 123, no. 8, pp. 1930-1935, 2011.

[6] D. Rus and M. T. Tolley, "Design, fabrication and control of soft robots," Nature, vol. 521, no. 7553 , pp. $467-475,2015$.

[7] B. Mosadegh, P. Polygerinos, C. Keplinger, S. Wennstedt, R. F. Shepherd, U. Gupta, J. Shim, K. Bertoldi, C. J. Walsh, and G. M. Whitesides, "Pneumatic networks for soft robotics that actuate rapidly," Advanced functional materials, vol. 24, no. 15, pp. 2163-2170, 2014.

[8] W. McMahan, B. A. Jones, and I. D. Walker, "Design and implementation of a multi-section continuum robot: Air-octor," in Intelligent Robots and Systems, 2005.(IROS 2005). 2005 IEEE/RSJ International Conference on, pp. 2578-2585, IEEE, 2005.

[9] R. K. Katzschmann, A. D. Marchese, and D. Rus, "Hydraulic autonomous soft robotic fish for 3d swimming," in Experimental Robotics, pp. 405-420, Springer, 2016. 
[10] E. D. Engeberg, S. Dilibal, M. Vatani, J.-W. Choi, and J. Lavery, "Anthropomorphic finger antagonistically actuated by sma plates," Bioinspiration \& biomimetics, vol. 10, no. 5, p. $056002,2015$.

[11] E. Steltz, A. Mozeika, J. Rembisz, N. Corson, and H. Jaeger, "Jamming as an enabling technology for soft robotics," in Electroactive Polymer Actuators and Devices (EAPAD) 2010, vol. 7642, p. 764225, International Society for Optics and Photonics, 2010.

[12] M. Manti, V. Cacucciolo, and M. Cianchetti, "Stiffening in soft robotics: A review of the state of the art," IEEE Robotics \& Automation Magazine, vol. 23, no. 3, pp. 93-106, 2016.

[13] X. Zhou, C. Majidi, and O. M. OâĂŹReilly, "Soft hands: an analysis of some gripping mechanisms in soft robot design," International Journal of Solids and Structures, vol. 64, pp. 155-165, 2015.

[14] R. Pfeifer, F. Iida, and M. Lungarella, "Cognition from the bottom up: on biological inspiration, body morphology, and soft materials," Trends in cognitive sciences, vol. 18, no. 8, pp. 404-413, 2014.

[15] M. Manti, T. Hassan, G. Passetti, N. D’Elia, C. Laschi, and M. Cianchetti, "A bioinspired soft robotic gripper for adaptable and effective grasping," Soft Robotics, vol. 2, no. 3, pp. 107$116,2015$.

[16] L. U. Odhner, L. P. Jentoft, M. R. Claffee, N. Corson, Y. Tenzer, R. R. Ma, M. Buehler, R. Kohout, R. D. Howe, and A. M. Dollar, "A compliant, underactuated hand for robust manipulation," The International Journal of Robotics Research, vol. 33, no. 5, pp. 736-752, 2014.

[17] C. Eppner, R. Deimel, J. Álvarez-Ruiz, M. Maertens, and O. Brock, "Exploitation of environmental constraints in human and robotic grasping," The International Journal of Robotics Research, vol. 34, no. 7, pp. 1021-1038, 2015. 
[18] E. Brown, N. Rodenberg, J. Amend, A. Mozeika, E. Steltz, M. R. Zakin, H. Lipson, and H. M. Jaeger, "Universal robotic gripper based on the jamming of granular material," Proceedings of the National Academy of Sciences, vol. 107, no. 44, pp. 18809-18814, 2010.

[19] A. Verl, A. Albu-Schäffer, O. Brock, and A. Raatz, Soft robotics: Transferring theory to application. Springer, 2015.

[20] M. Giorelli, F. Renda, M. Calisti, A. Arienti, G. Ferri, and C. Laschi, "A two dimensional inverse kinetics model of a cable driven manipulator inspired by the octopus arm," in Robotics and Automation (ICRA), 2012 IEEE International Conference on, pp. 3819-3824, IEEE, 2012.

[21] A. Shiva, A. Stilli, Y. Noh, A. Faragasso, I. De Falco, G. Gerboni, M. Cianchetti, A. Menciassi, K. Althoefer, and H. A. Wurdemann, “Tendon-based stiffening for a pneumatically actuated soft manipulator," IEEE Robotics and Automation Letters, vol. 1, no. 2, pp. 632637, 2016.

[22] F. Maghooa, A. Stilli, Y. Noh, K. Althoefer, and H. A. Wurdemann, "Tendon and pressure actuation for a bio-inspired manipulator based on an antagonistic principle," in Robotics and Automation (ICRA), 2015 IEEE International Conference on, pp. 2556-2561, IEEE, 2015.

[23] A. Stilli, H. A. Wurdemann, and K. Althoefer, "Shrinkable, stiffness-controllable soft manipulator based on a bio-inspired antagonistic actuation principle," in Intelligent Robots and Systems (IROS 2014), 2014 IEEE/RSJ International Conference on, pp. 2476-2481, IEEE, 2014.

[24] A. Jiang, E. Secco, H. Wurdemann, T. Nanayakkara, P. Dasgupta, and K. Athoefer, "Stiffnesscontrollable octopus-like robot arm for minimally invasive surgery," in Workshop on New Technologies for Computer/Robot Assisted Surgery, 2013.

[25] M. Li, T. Ranzani, S. Sareh, L. D. Seneviratne, P. Dasgupta, H. A. Wurdemann, and K. Althoefer, "Multi-fingered haptic palpation utilizing granular jamming stiffness feedback actuators," Smart Materials and Structures, vol. 23, no. 9, p. 095007, 2014. 
[26] Y.-J. Kim, S. Cheng, S. Kim, and K. Iagnemma, "Design of a tubular snake-like manipulator with stiffening capability by layer jamming," in Intelligent Robots and Systems (IROS), 2012 IEEE/RSJ International Conference on, pp. 4251-4256, IEEE, 2012.

[27] N. G. Cheng, A. Gopinath, L. Wang, K. Iagnemma, and A. E. Hosoi, “Thermally tunable, self-healing composites for soft robotic applications," Macromolecular Materials and Engineering, vol. 299, no. 11, pp. 1279-1284, 2014.

[28] A. A. Stokes, R. F. Shepherd, S. A. Morin, F. Ilievski, and G. M. Whitesides, "A hybrid combining hard and soft robots," Soft Robotics, vol. 1, no. 1, pp. 70-74, 2014.

[29] M. Li, R. Kang, S. Geng, and E. Guglielmino, "Design and control of a tendon-driven continuum robot," Transactions of the Institute of Measurement and Control, vol. 0, no. 0, p. 0142331216685607,0 .

[30] H. Liu and J. Lee, "Model development and experimental research on an air spring with auxiliary reservoir," International Journal of Automotive Technology, vol. 12, no. 6, pp. 839$847,2011$.

[31] Z. Sihong, W. Jiasheng, and Z. Ying, "Research on theoretical calculation model for dynamic stiffness of air spring with auxiliary chamber," in Vehicle Power and Propulsion Conference, 2008. VPPC'08. IEEE, pp. 1-6, IEEE, 2008.

[32] B. W. Anderson, The analysis and design of pneumatic systems. Wiley, 1967.

[33] A. D. Marchese and D. Rus, "Design, kinematics, and control of a soft spatial fluidic elastomer manipulator," The International Journal of Robotics Research, vol. 35, no. 7, pp. 840$869,2016$.

[34] A. Fakhari, M. Keshmiri, I. Kao, and S. Hadian Jazi, "Slippage control in soft finger grasping and manipulation," Advanced Robotics, vol. 30, no. 2, pp. 97-108, 2016.

[35] H.-Y. Han, A. Shimada, and S. Kawamura, "Analysis of friction on human fingers and design of artificial fingers," in Robotics and Automation, 1996. Proceedings., 1996 IEEE International Conference on, vol. 4, pp. 3061-3066, IEEE, 1996. 
[36] F. Ficuciello, Modelling and Control for Soft Finger Manipulation and Human-Robot Interaction. PhD thesis, Università degli Studi di Napoli Federico II, 2010.

[37] M. Peters, K. Mackenzie, and P. Bryden, "Finger length and distal finger extent patterns in humans," American journal of physical anthropology, vol. 117, no. 3, pp. 209-217, 2002. 